TRANSACTIONS OF THE

AMERICAN MATHEMATICAL SOCIETY

Volume 364, Number 12, December 2012, Pages 6229-6258

S 0002-9947(2012)05440-7

Article electronically published on July 11, 2012

\title{
LAWSON CORRESPONDENCE AND RIBAUCOUR TRANSFORMATIONS
}

\author{
M. LEMES, P. ROITMAN, K. TENENBLAT, AND R. TRIBUZY
}

\begin{abstract}
We prove that Darboux transformations commute with the Lawson correspondence and we show that the property of completeness is preserved by this commutativity. We provide examples of these results. Two applications provide families of explicitly parametrized complete surfaces of constant mean curvature 1 and $-\sqrt{5} / 2$ in $\mathbb{H}^{3}$, depending on 2 parameters and 1 parameter respectively. For special choices of the parameters, we get surfaces that are periodic in one variable and in particular complete cmc surfaces or cmc1 surfaces in $\mathbb{H}^{3}$, with any finite or infinite number of bubbles, "segments" or embedded ends of horosphere type. Moreover, we consider Ribaucour transformations for associated linear Weingarten surfaces in space forms. We show that such a transformation is a Darboux transformation (i.e., it is conformal) if and only if the surfaces have the same constant mean curvature. We prove that Ribaucour transformations for surfaces with constant mean curvature 1 ( $\mathrm{cmc} 1)$ immersed in the hyperbolic space $\mathbb{H}^{3}$ produce embedded ends of horosphere type.
\end{abstract}

\section{INTRODUCTION}

Ribaucour transformations were classically studied by Bianchi Bi, Bi2 to obtain new surfaces of constant Gaussian curvature from a given such surface. Although Ribaucour transformations for minimal surfaces were also classically known, the first examples were given in CFT1. Ribaucour transformations were extended to linear Weingarten surfaces in [CFT2, [TW1 and applied to produce families of cmc surfaces in space forms.

The Lawson correspondence La associates isometric surfaces $M$ and $M^{\prime}$ of distinct constant mean curvature $H$ and $H^{\prime}$, immersed in space forms of constant sectional curvature $k$ and $k^{\prime}$ respectively, such that $k+H^{2}=k^{\prime}+\left(H^{\prime}\right)^{2}$. Whenever $M$ is a minimal surface, then $M^{\prime}$ is also referred to as a cmc cousin of $M$.

In this paper, we prove some properties of Ribaucour transformations, relate these transformations to the Lawson correspondence and provide some applications.

In general, a Ribaucour transformation between linear Weingarten surfaces is not a Darboux transformation, as was shown in CFT2. We show that the Ribaucour transformations between linear Weingarten surfaces which are conformal (hence Darboux transformations) are precisely those transformations for surfaces of the same constant mean curvature (Theorem 2.1).

Moreover, we prove that Ribaucour transformations for cmc1 surfaces in the hyperbolic space $\mathbb{H}^{3}$ produce embedded ends of horosphere type (Theorem 2.2).

Received by the editors March 12, 2010 and, in revised form, July 30, 2010.

2010 Mathematics Subject Classification. Primary 53C20.

Key words and phrases. Ribaucour transformations, constant mean curvature surfaces, Lawson correspondence, Darboux transformations, horosphere type end.

This work was partially supported by CNPq, CAPES/PROCAD. 
In [CFT1, such transformations for minimal surfaces in the Euclidean space were shown to produce planar embedded ends.

In Theorem 3.1 we prove that the Lawson correspondence $\mathrm{La}$ ] commutes with the Darboux transformation (or Ribaucour transformation for cmc surfaces). Although this result was known to Bianchi [Bi1] (see also [HJ]), our proof is very useful for the applications in section 4 and it also allows us to show in Corollary 3.2 that the property of completeness is preserved by this commutativity.

We illustrate Theorem 3.1 with the following examples of parametrized surfaces: The cmc1 surfaces in $\mathbb{R}^{3}$ associated to the cylinder by Ribaucour transformations CFT1 and the minimal complete surfaces in $S^{3}$ associated to the Clifford torus TW1 are related by the Lawson correspondence. The family of complete minimal surfaces in $\mathbb{R}^{3}$ associated to the catenoid (resp. Enneper surface) by Ribaucour transformations obtained in CFT1 and the family of complete cmc1 surfaces in $\mathbb{H}^{3}$ associated to a catenoid cousin (resp. Enneper's cousin) obtained in TW2] are in correspondence as in Theorem 3.1.

We provide two applications of Theorem 3.1. In the first one, we consider a Bonnet family of minimal surfaces in $\mathbb{R}^{3}$ and their cmc1 cousins in $\mathbb{H}^{3}$. Since the minimal surfaces in $\mathbb{R}^{3}$ associated to the Bonnet family by Ribaucour transformations were obtained in $[\mathrm{LT}$, we then obtain families of complete parametrized cmc1 surfaces in $\mathbb{H}^{3}$ (Theorem 4.1 and Theorem 4.3).

This family includes explicitly given parametrized cmc1 surfaces associated to cousins of the catenoid, depending on 2 parameters. According to the choice of one of the parameters, we produce surfaces that have infinitely many embedded ends of horosphere type or periodic surfaces in one variable that have any fixed number of embedded ends of horosphere type. Figure 2 exhibits a cmc1 surface associated to the singular cousin of the catenoid, and Figures 4-16 provide illustrations of cmc1 surfaces associated to an embedded cousin of the catenoid. Figures 18 and 19 exhibit a cmc1 surface associated to a nonembedded cousin of the catenoid obtained by Umehara-Yamada [UY].

In the second application, we consider the cylinder of radius one in $\mathbb{R}^{3}$ and its cmc cousin in $\mathbb{H}^{3}$, with $H=-\sqrt{5} / 2$. By using the cmc surfaces associated to the cylinder by Ribaucour transformations, we then obtain a 1-parameter family of explicitly parametrized complete surfaces, with mean curvature $H=-\sqrt{5} / 2$ in $\mathbb{H}^{3}$ (Theorem 4.5). Moreover, according to the choice of this parameter, we produce surfaces that have infinitely many bubbles or "segments" or periodic surfaces in one variable that have any fixed number of bubbles or "segments". Figures 21-32 exhibit surfaces of this family.

We should mention that surfaces in Euclidean 3 -space $\mathbb{R}^{3}$ with bubbles have been studied by Sterling-Wente [SW]. Such a surface was first found by Sievert [Si]. In CFT2, a large family of cmc cylinders with bubbles and Delaunay surfaces with bubbles in $\mathbb{R}^{3}$ were obtained by using the Ribaucour transformation. These families include not only surfaces with a finite number of bubbles (in this case the surfaces are periodic in one variable), but also an infinite number of bubbles around the axis of rotation of the Delaunay surface or in the direction perpendicular to this axis. Cylinders with a finite or an infinite number of bubbles in $S^{3}$ were obtained in TW1.

We observe that, for a general cmc surface in $\mathbb{R}^{3}$, Burstall $[\mathrm{Bu}]$ showed that simple type dressings for real and pure imaginary parameters are equivalent to Darboux 
transformations. Hetrich-Jeromin and Pedit [HJP] proved that a Bianchi-Bäcklund transformation of a cmc surface in $\mathbb{R}^{3}$ is necessarily a Darboux transformation, but not the converse. Kobayashi $[\mathrm{Ko}$ showed that when one considers a round cylinder in $\mathbb{R}^{3}$, then the Bianchi-Bäcklund transformation is equivalent to a simple type dressing and hence to a Darboux transformation. We observe that cylinder bubbletons with cmc1 in $\mathbb{H}^{3}$ and cmc0 in the sphere $S^{3}$ were constructed by Kobayashi, by numerical methods, using the DPW method [DPW]. These were also constructed by Schmitt $[\mathrm{Sc}$ using CMCLab software.

In contrast to these numerical methods, all the families of cmc surfaces in space forms given in this paper and in the previous ones ([CFT1, [CFT2, [TW1], TW2]) are described by explicit parametrizations, obtained by Ribaucour transformations. The parametrizations were also used to visualize all the surfaces.

In section 1, we recall the main results on Ribaucour transformations for linear Weingarten surfaces in space forms. In section 2, we obtain two properties of the Ribaucour transformations. Namely, we show that the only Ribaucour transformations for linear Weingarten surfaces which are Darboux transformations are those for surfaces with the same constant mean curvature. Moreover, we prove that Ribaucour transformations for cmc1 surfaces in $\mathbb{H}^{3}$ produce embedded ends of horosphere type.

In section 3, we prove that the Lawson correspondence commutes with the Darboux transformation. We also show that the property of completeness is preserved by this commutativity. The applications of these results are given in section 4 .

\section{Ribaucour transformations For LineAR WEINGARTEn SURFACES IN SPACE FORMS}

In this section, we recall the main results that will be used in the following sections; namely, the fact that Ribaucour transformations provide an integrable system of differential equations whose solutions enable us to obtain linear Weingarten surfaces in a space form $\bar{M}^{3}$ from a given such surface and in particular cmc surfaces from a given such surface. For more details and proofs, see [TW1] and its references.

Let $\bar{M}^{3}(k), k= \pm 1,0$, be the simply connected space form of sectional curvature $k$ and let $\mathbb{L}^{4}$ be the set of points $x=\left(x_{0}, x_{1}, \ldots, x_{3}\right) \in \mathbb{R}^{4}$ endowed with the pseudoRiemannian inner product given by $\langle x, y\rangle=-x_{0} y_{0}+\sum_{i=1}^{3} x_{i} y_{i}$. We consider the hyperbolic three-space as the submanifold of $\mathbb{L}^{4}, \mathbb{H}^{3}=\left\{x \in \mathbb{L}^{4} \mid\langle x, x\rangle=-1\right\}$, with two connected components. It will be useful in section 4 .

Consider an orientable surface $M$ in $\bar{M}^{3}(k)$, where $\bar{M}^{3}(k)=\mathbb{R}^{3}$ when $k=0$, $\bar{M}^{3}(k)=S^{3} \subset \mathbb{R}^{4}$ when $k=1$ and $\bar{M}^{3}(k)=\mathbb{H}^{3} \subset \mathbb{L}^{4}$ when $k=-1$. Let $e_{i}, i=1,2$, be an orthonormal frame tangent to $M$ and $N$ a unit normal vector field defined on $M$. Denote by $\omega_{i}$ the 1 -forms dual to $e_{i}$ and $\omega_{i j}, i, j \in\{1,2\}$, the connection forms which are defined by $d \omega_{i}=\sum_{j \neq i} \omega_{i j} \wedge w_{j}, \omega_{i j}+\omega_{j i}=0$. The Gauss equation is given by $d \omega_{12}=\omega_{13} \wedge \omega_{32}-k \omega_{1} \wedge \omega_{2}$, where $\omega_{i 3}=-\omega_{3 i}=\left\langle d e_{i}, N\right\rangle$, and the Codazzi equations are $d \omega_{i 3}=\sum_{j=1}^{2} \omega_{i j} \wedge \omega_{j 3}$. If the vector fields $e_{i}$ are the principal directions corresponding to the principal curvatures $-\lambda_{i}, i=1,2$, then

$$
\omega_{i 3}=-\lambda_{i} \omega_{i}, \quad d N\left(e_{i}\right)=\lambda_{i} e_{i}
$$


An orientable surface $\tilde{M} \subset \bar{M}^{3}(k)$ is associated to $M$ by a Ribaucour transformation if there exist a differentiable function $h: M \rightarrow \mathbb{R}$, a diffeomorphism $\psi: M \rightarrow \tilde{M}$ and unit normal vector fields $N$ and $\tilde{N}$ of $M$ and $\tilde{M}$, respectively, such that

a) $\exp _{p} h(p) N(p)=\exp _{\psi(p)} h(p) \tilde{N}(\psi(p)), \forall p \in M$, where exp is the exponential map of $\bar{M}$;

b) the subset $M_{0}=\left\{\exp _{p} h(p) N(p) \mid p \in M\right\}$ is a surface contained in $\bar{M}$;

c) $d \psi\left(e_{i}\right), i=1,2$, are orthogonal principal directions of $\tilde{M}$.

Condition a) can be rewritten as

$$
p+h(p) N(p)=\psi(p)+h(p) \tilde{N}(\psi(p)), \quad p \in M, \text { if } k=0,
$$

and

$$
\begin{array}{cl}
p+\tan (h(p)) N(p)=\psi(p)+\tan (h(p)) \tilde{N}(\psi(p)) & \text { if } k=1, \\
p+\tanh (h(p)) N(p)=\psi(p)+\tanh (h(p)) \tilde{N}(\psi(p)) & \text { if } k=-1 .
\end{array}
$$

One can also formulate a definition for surfaces locally associated by Ribaucour transformations.

A characterization of a Ribaucour transformation in a space form is given in terms of a system of nonlinear partial differential equations for $h$. However, the problem of obtaining $h$ can be linearized by considering $h=\Omega / W$. The following theorem provides a sufficient condition on $\Omega$ and $W$ for a Ribaucour transformation to transform a linear Weingarten surface in a space form into another surface of the same type. We refer to [CFT2 and TW1 for the proofs and details.

Theorem 1.1 (CFT2, TW1]). Let $M$ be a linear Weingarten surface in $\bar{M}^{3}(k)$, which admits the orthonormal principal vector fields $e_{1}$ and $e_{2}$. Suppose that the Gaussian curvature $K$ and the mean curvature $H$ of $M$ satisfy $\alpha+\beta H+\gamma(K-k)=$ 0 . Then for any constant $c \neq 0$, the system of the equations

$$
\begin{aligned}
& d \Omega=\sum_{i=1}^{2} \Omega_{i} \omega_{i}, \\
& d W=\sum_{i=1}^{2} \Omega_{i} \omega_{i 3}, \\
& d \Omega_{i}=\Omega_{j} \omega_{i j}+\{(2 c \alpha-k) \Omega+\beta c W\} \omega_{i}+\{c \beta \Omega+(2 c \gamma-1) W\} \omega_{i 3}, i \neq j
\end{aligned}
$$

is integrable. Any solution of (11), on a simply connected domain, whose initial condition satisfies

$$
\Omega_{1}^{2}+\Omega_{2}^{2}+W^{2}+k \Omega^{2}=2 c\left(\alpha \Omega^{2}+\beta \Omega W+\gamma W^{2}\right)
$$

will satisfy (2) identically. If $M$ is locally parametrized by $X: U \subset \mathbb{R}^{2} \rightarrow M \subset$ $\bar{M}^{3}(k)$, and $\Omega, W$ is a nontrivial solution of (1) satisfying (2), then each surface of the family

$$
\widetilde{X}=\left(1-\frac{2 k \Omega^{2}}{S}\right) X-\frac{2 \Omega}{S}\left(\sum_{i=1}^{2} \Omega_{i} e_{i}-W N\right),
$$

where

$$
S=\Omega_{1}^{2}+\Omega_{2}^{2}+W^{2}+k \Omega^{2},
$$


is a linear Weingarten surface satisfying $\alpha+\beta \widetilde{H}+\gamma(\widetilde{K}-k)=0$, where $\widetilde{H}$ and $\widetilde{K}$ are the mean and Gaussian curvature of $\widetilde{X}$ respectively. Its unit normal vector field is given by

$$
\widetilde{N}=\left(1-\frac{2 W^{2}}{S}\right) N+\frac{2 W}{S}\left(\sum_{i=1}^{2} \Omega_{i} e_{i}+k \Omega X\right) .
$$

Moreover, each surface described by $\widetilde{X}$ is locally associated to $X$ by a Ribaucour transformation and it is defined on

$$
\widetilde{U}=\left\{\left(u_{1}, u_{2}\right) \in U: S\left(T^{2}+2 T Q H+Q^{2}(K-k)\right) \neq 0\right\}
$$

where $T=\alpha \Omega^{2}-\gamma W^{2}$ and $Q=2 \gamma \Omega W+\beta \Omega^{2}$.

One can show that the first fundamental form of $\tilde{X}$ is given by $\tilde{I}=\tilde{\omega}_{1}^{2}+\tilde{\omega}_{2}^{2}$, where

$$
\tilde{\omega}_{i}=\frac{\gamma W^{2}-\alpha \Omega^{2}+(\beta \Omega+2 \gamma W) \Omega \lambda_{i}}{\alpha \Omega^{2}+\beta \Omega W+\gamma W^{2}} \omega_{i}, \quad i=1,2,
$$

and the principal curvatures $-\tilde{\lambda}_{i}$ are defined by

$$
\tilde{\lambda}_{i}=\frac{2 \alpha \Omega W+\beta W^{2}+\lambda_{i}\left(\alpha \Omega^{2}-\gamma W^{2}\right)}{\left(2 \gamma \Omega W+\beta \Omega^{2}\right) \lambda_{i}-\left(\alpha \Omega^{2}-\gamma W^{2}\right)}, \quad i=1,2 .
$$

By considering $H$ constant, i.e., $\alpha=-H, \beta=1$ and $\gamma=0$ in Theorem 1.1, we have the Ribaucour transformation for $\mathrm{cmcH}$ surfaces $(H=0$ is the case for minimal surfaces), given by the following theorem:

Theorem 1.2. Let $M$ be a cmcH surface in $\bar{M}^{3}(k)$ with no umbilic points and let $e_{1}, e_{2}$ be orthonormal principal directions. For any constant $c \neq 0$ the system of equations

$$
\begin{aligned}
& d \Omega=\sum_{i=1}^{2} \Omega_{i} \omega_{i}, \\
& d W=\sum_{i=1}^{2} \Omega_{i} \omega_{i 3}, \\
& d \Omega_{i}=\Omega_{j} \omega_{i j}-[(2 c H+k) \Omega-c W] \omega_{i}+(c \Omega-W) \omega_{i 3}, i \neq j
\end{aligned}
$$

is integrable. Assume that

$$
-k+c^{2}-2 H c>0 .
$$

Then any solution of (6) on a simply connected domain, whose initial condition satisfies

$$
S=2 c \Omega(W-H \Omega),
$$

where $S$ is given by (3), will satisfy (8) identically. If $M$ is locally parametrized by $X: U \subset \mathbb{R}^{2} \rightarrow \bar{M}^{3}(k)$, and $\Omega, W$ is a solution of (6) satisfying (7) and (8)), then each surface of the family

$$
\widetilde{X}=\left(1-\frac{k \Omega}{c(W-H \Omega)}\right) X-\frac{1}{c(W-H \Omega)}\left(\sum_{i=1}^{2} \Omega_{i} e_{i}-W N\right)
$$


is a cmcH surface locally associated to $X$ by a Ribaucour transformation defined on the subset of $U$ where $\Omega(W-H \Omega) \neq 0$. Moreover, the normal vector field of $\widetilde{X}$ is given by

$$
\widetilde{N}=\left(1-\frac{W^{2}}{c \Omega(W-H \Omega)}\right) N+\frac{W}{c \Omega(W-H \Omega)}\left(\sum_{i=1}^{2} \Omega_{i} e_{i}+k \Omega X\right) .
$$

We observe that (7) is a necessary condition for a nontrivial solution to satisfy (8). Moreover, the first fundamental form of $\tilde{X}$ is given by $\tilde{I}=\tilde{\omega}_{1}^{2}+\tilde{\omega}_{2}^{2}$, where

$$
\tilde{\omega}_{i}=\frac{\Omega\left(H+\lambda_{i}\right)}{W-H \Omega} \omega_{i}, \quad i=1,2,
$$

and the principal curvatures $-\tilde{\lambda}_{i}$ are defined by

$$
\tilde{\lambda}_{i}=\frac{(W-H \Omega)^{2}}{\Omega^{2}\left(H+\lambda_{i}\right)}-H, \quad i=1,2 .
$$

One observes that given a cmcH surface, a Ribaucour transformation, generically, provides (locally) a 3-parameter family of $\mathrm{cmcH}$ surfaces associated to the given surface.

For later use, by considering a surface parametrized by lines of curvature $X\left(u_{1}, u_{2}\right)$, we rewrite the integrable system (6) as a system of differential equations for $\Omega, \Omega_{i}, W$ as follows:

$$
\begin{aligned}
& \frac{\partial \Omega}{\partial u_{i}}=a_{i} \Omega_{i}, \quad \frac{\partial W}{\partial u_{i}}=-\lambda_{i} \frac{\partial \Omega}{\partial u_{i}}, \\
& \frac{\partial \Omega_{i}}{\partial u_{j}}=\frac{\Omega_{j}}{a_{i}} \frac{\partial a_{j}}{\partial u_{i}}, \quad i \neq j, \\
& \frac{\partial \Omega_{i}}{\partial u_{i}}=-\frac{\Omega_{j}}{a_{j}} \frac{\partial a_{i}}{\partial u_{j}}-\left[c\left(2 H+\lambda_{i}\right)+k\right] a_{i} \Omega+\left(c+\lambda_{i}\right) a_{i} W .
\end{aligned}
$$

In general, a Ribaucour transformation between linear Weingarten surfaces, given by Theorem 1.1, is not a Darboux transformation (see for example [CFT2], p. 278). In the next section, we will show that the Ribaucour transformations between linear Weingarten surfaces which are conformal (hence Darboux transformations) are precisely those transformations for surfaces of the same constant mean curvature.

\section{Properties of the Ribaucour transformation}

In this section, we obtain two properties of the Ribaucour transformations. Namely, we show that the only Ribaucour transformations for linear Weingarten surfaces which are Darboux transformations are those for surfaces with the same constant mean curvature. Moreover, we prove that Ribaucour transformations for cmc1 surfaces in $\mathbb{H}^{3}$ produce embedded ends of horosphere type. We recall that such transformations for minimal surfaces in $\mathbb{R}^{3}$ were shown to produce embedded planar ends in CFT1.

Theorem 2.1. Let $M$ and $\tilde{M}$ be linear Weingarten surfaces in $\bar{M}^{3}(k)$ associated by a Ribaucour transformation as in Theorem 1.1. Then the transformation is conformal if and only if $M$ and $\tilde{M}$ have the same constant mean curvature. 
Proof. If $M$ and $\tilde{M}$ have the same constant mean curvature, it follows from (11) that the associated surfaces are conformal and hence the Ribaucour transformation is a Darboux transformation.

Conversely, assume that the linear Weingarten surfaces, associated by a Ribaucour transformation, are conformal. Then it follows from (5) that

$$
(\beta \Omega+2 \gamma W) \Omega\left(\lambda_{1}-\lambda_{2}\right) \gamma W^{2}-\alpha \Omega^{2}-(\beta \Omega+2 \gamma W) \Omega H=0 .
$$

Therefore on each open subset $V$ of $M$ either

$$
(\beta \Omega+2 \gamma W) \Omega\left(\lambda_{1}-\lambda_{2}\right)=0
$$

or

$$
\gamma W^{2}-\alpha \Omega^{2}-(\beta \Omega+2 \gamma W) \Omega H=0
$$

In each case, assuming that $H$ is not constant on $M$, we will obtain a contradiction.

Assume (14) occurs. Since $\Omega \not \equiv 0$ on $V$, then either $\lambda_{1}-\lambda_{2} \equiv 0$ or $\beta \Omega+2 \gamma W \equiv 0$. If $\lambda_{1}-\lambda_{2} \equiv 0$, then $H=-\lambda_{1}$ and $\alpha+\beta H+\gamma(K-k)=0$; i.e., $\alpha-\beta \lambda_{1}+\gamma\left(\lambda_{1}\right)^{2}=0$. Therefore, $\lambda_{1}$ and hence $H$ is constant on $V$. If $\beta \Omega+2 \gamma W=0$ on an open subset $V$ of $M$, we have $\beta \neq 0$, since otherwise we would have $\gamma W=0$. Therefore, $h=\Omega / W=-2 \gamma / \beta$ and $\beta d \Omega+2 \gamma d W=0$. It follows from (11) that $\Omega_{i}\left(\beta-2 \gamma \lambda_{i}\right)=0$ on $V$, for $i=1,2$. Observe that $1+h \lambda_{j} \neq 0$ for all $j$. In fact, since the subset $M_{0}$ of a Ribaucour transformation is a surface, the functions $1+h \lambda_{j}, j=1,2$, do not vanish (see CFT1, TW1]). It follows that we have $\beta-2 \gamma \lambda_{i} \neq 0$. Therefore, $\Omega_{i}=0$ for all $i$. Hence $\Omega$ and $W$ are constant and it follows from the third equation of (11) that

$$
0=(2 c \alpha-k) \Omega-\beta c W-\lambda_{i} W, \quad i=1,2 .
$$

We conclude that $\lambda_{1}=\lambda_{2}$ is a constant and $H$ is constant, which is a contradiction.

If (15) occurs, then

$$
-(\alpha+\beta H) \Omega^{2}+\gamma\left(W^{2}-2 W \Omega H\right)=0 .
$$

Since $M$ satisfies $\alpha+\beta H+\gamma(K-k)=0$, and $\gamma \neq 0$, we have that

$$
W^{2}+(K-k) \Omega^{2}-2 W \Omega H=0
$$

i.e., $\left(W+\Omega \lambda_{1}\right)\left(W+\Omega \lambda_{2}\right)=0$. This is equivalent to $\left(1+h \lambda_{1}\right)\left(1+h \lambda_{2}\right)=0$, which is a contradiction.

We conclude that $H$ is constant and $\tilde{H}=H$.

In CFT2, it was shown that Ribaucour transformations for minimal surfaces produce embedded planar ends. In our next result, we show that Ribaucour transformations for cmc1 surfaces in the hyperbolic three-dimensional space produce embedded ends of horosphere type.

An end of a cmc1 surface in $\mathbb{H}^{3}$ is called regular if the hyperbolic Gauss map of the surface has no essential singularity at the end. Otherwise, the end is called irregular. Results of Earp-Toubiana [ET] and Lima-Rossman [LR] prove that a regular end with finite total curvature is proper and it is asymptotic to a catenoid cousin or a horosphere. We will show that Ribaucour transformations for cmc1 surfaces in $\mathbb{H}^{3}$ produce ends that are asymptotic to horospheres.

Let $X: M \rightarrow \mathbb{H}^{3}$ be an immersion of a cmc1 surface. The unit normal vector $N$ of $X$ is a map from $M$ into the De Sitter space

$$
S_{1}^{3}=\left\{\mathbf{x} \in \mathbb{L}^{4}:\langle\mathbf{x}, \mathbf{x}\rangle=1\right\}
$$


in such a way that if $u_{1}, u_{2}$ are the parameters of $M$, then $\left\{X, X_{u_{1}}, X_{u_{2}}, N\right\}$ is a positively oriented basis of $\mathbb{L}^{4}$. The map $X+N: M \rightarrow \mathbb{L}^{4}$ takes its values in the positive light cone

$$
\mathbb{N}^{3}=\left\{\mathbf{x} \in \mathbb{L}^{4}:\langle\mathbf{x}, \mathbf{x}\rangle=0, x_{0}>0\right\} .
$$

By projecting $\mathbb{N}^{3}$ into the quotient $\mathbb{N}^{3} / \mathbb{R}^{+}$, one obtains a map $G=[X+N]: M \rightarrow$ $\partial \mathbb{H}^{3}$, which is called the hyperbolic Gauss map of $X$. The geometric interpretation of $G$ is the following: for each point $\left(u_{1}, u_{2}\right)$ of $M$, the oriented normal geodesic starting from $X\left(u_{1}, u_{2}\right)$ meets the ideal boundary $\partial \mathbb{H}^{3}$ of $\mathbb{H}^{3}$ at $G\left(u_{1}, u_{2}\right)$.

We now consider a Ribaucour transformation for surfaces $X$ and $\widetilde{X}$ as in Theorem 1.1. Assume that $p_{0}$ is a point that annihilates $S$, where $S$ is given by (3). We want to find out the behavior of $\widetilde{X}$ in a neighborhood of $p_{0}$. For minimal surfaces in $\mathbb{R}^{3}$, one can show (see [CFT1]) that $\lim _{p \rightarrow p_{0}} \widetilde{N}(p)=N\left(p_{0}\right)$ and each $p_{0}$ generates an embedded planar end on the surface $\tilde{X}$. In the case of cmc1 surfaces in $\mathbb{H}^{3}$, one has a similar behavior, replacing the unit normal vector by the hyperbolic Gauss map. This is the content of our next theorem, where we also show that $p_{0}$ produces an embedded horosphere type end. We should mention that the first results in this direction were obtained in TW2 for the family of surfaces associated to an Enneper cousin and to the singular catenoid cousin.

Theorem 2.2. Let $\widetilde{X}: D \backslash\left\{p_{0}\right\} \subset \mathbb{R}^{2} \rightarrow \mathbb{H}^{3}$ be a cmc1 surface, locally associated by a Ribaucour transformation to a cmc1 surface $X: D \subset \mathbb{R}^{2} \rightarrow \mathbb{H}^{3}$ such that the functions $\Omega_{i}, \Omega$ and $W$ are defined on $D$. Let $\widetilde{G}$ and $G$ be the hyperbolic Gauss maps of $\widetilde{X}$ and $X$, respectively. If $S\left(p_{0}\right)=0, \Omega\left(p_{0}\right) \neq 0$ and $S(p) \neq 0$ for all $p \in D \backslash\left\{p_{0}\right\}$, then the cmc1 surface $\widetilde{X}$ has an embedded horosphere type end at $p_{0}$, and $\lim _{p \rightarrow p_{0}} \widetilde{G}(p)=G\left(p_{0}\right)$.

Proof. Since $\widetilde{X}$ is a cmc1 surface, one can assume that in local coordinates the first and second fundamental forms of $\widetilde{X}$ are given by

$$
\tilde{I}=\varphi^{2}\left(d u_{1}^{2}+d u_{2}^{2}\right) \quad \text { and } \quad \tilde{I} I=-2 \nu\left(d u_{1}^{2}-d u_{2}^{2}\right)+I,
$$

where $I$ is the first fundamental form of $X$ and

$$
\left(1+\lambda_{i}\right) \varphi^{2}=(-1)^{i+1} 2 \nu
$$

It follows from (9) and (10) that

$$
\tilde{G}=\widetilde{X}+\widetilde{N}=\left(1-\frac{1}{c}\right) X+\left(1-\frac{W}{c \Omega}\right) N+\frac{1}{c \Omega}\left(\Omega_{1} e_{1}+\Omega_{2} e_{2}\right) .
$$

From (8), since $S=2 c \Omega(W-\Omega), S\left(p_{0}\right)=0$ and $\Omega\left(p_{0}\right) \neq 0$, it follows that

$$
W\left(p_{0}\right)=\Omega\left(p_{0}\right) .
$$

Moreover, it follows from (3) that

$$
\Omega_{1}^{2}\left(p_{0}\right)+\Omega_{2}^{2}\left(p_{0}\right)=0 .
$$

Hence,

$$
\lim _{p \rightarrow p_{0}}(\widetilde{X}+\widetilde{N})=\left(1-\frac{1}{c}\right)(X+N)\left(p_{0}\right)
$$

and

$$
\lim _{p \rightarrow p_{0}} \widetilde{G}(p)=G\left(p_{0}\right) .
$$


In order to show that $p_{0}$ is an embedded horosphere type end, we consider $\widetilde{X}$ given by (9) in the upper half space model, i.e.,

$$
Z=\left(Z^{1}, Z^{2}, Z^{3}\right)=\left(\frac{\widetilde{X}^{1}}{\widetilde{X}^{0}-\widetilde{X}^{3}}, \frac{\widetilde{X}^{2}}{\widetilde{X}^{0}-\widetilde{X}^{3}}, \frac{1}{\widetilde{X}^{0}-\widetilde{X}^{3}}\right)
$$

where $\widetilde{X}^{j}$ is the $j$-th coordinate function of $\widetilde{X}$. It follows from (9) that

$$
Z^{j}=\frac{V^{j}}{V^{0}-V^{3}}, \quad j=1,2, \quad Z^{3}=\frac{c(W-\Omega)}{V^{0}-V^{3}},
$$

where

$$
V=[c(W-\Omega)+\Omega+W] X-\Omega_{1} e_{1}+\Omega_{2} e_{2}
$$

and $V^{j}$ denotes the $j$-th coordinate of $V$.

If we consider $Y=X+N$, we have $V\left(p_{0}\right)=\Omega\left(p_{0}\right) Y\left(p_{0}\right)$. It follows from (6) that

$$
W_{, i}\left(p_{0}\right)=\Omega_{, i}\left(p_{0}\right)=0,
$$

where the lower index ", $i$ " means the partial derivative with respect to $u_{i}$ and

$$
\Omega_{i, i}\left(p_{0}\right)=(1-c)\left(1+\lambda_{i}\right) \varphi\left(p_{0}\right) \Omega\left(p_{0}\right) .
$$

Since $e_{i}=\frac{Y_{, i}}{\varphi\left(1+\lambda_{i}\right)}$, then $V_{, i}\left(p_{0}\right)=c \Omega\left(p_{0}\right) Y_{, i}\left(p_{0}\right)$; hence

$$
Z_{, i}^{j}\left(p_{0}\right)=c\left(\frac{Y^{j}}{Y^{0}-Y^{3}}\right)_{, i}\left(p_{0}\right)
$$

where $Y^{j}=X^{j}+N^{j}$.

The hyperbolic Gauss map in the upper half space model is given by

$$
G=\frac{Y^{1}+i Y^{2}}{Y^{0}-Y^{3}},
$$

where without loss of generality we are assuming that $p_{0}$ is not a pole of $G$. Moreover, $G$ is meromorphic and $Z_{, i}^{j}\left(p_{0}\right)=c G_{, i}^{j}\left(p_{0}\right), j=1,2$. For this reason we have

$$
\begin{array}{r}
Z_{, 1}^{1}\left(p_{0}\right)=c G_{, 1}^{1}\left(p_{0}\right)=c G_{, 2}^{2}\left(p_{0}\right)=Z_{, 2}^{2}\left(p_{0}\right), \\
Z_{, 1}^{2}\left(p_{0}\right)=c G_{, 1}^{2}\left(p_{0}\right)=-c G_{, 2}^{1}\left(p_{0}\right)=-Z_{, 2}^{1}\left(p_{0}\right) .
\end{array}
$$

It follows from (17) and (18) that $Z_{, i}^{3}\left(p_{0}\right)=0$ and

$$
Z_{, i j}^{3}\left(p_{0}\right)=\frac{c(W-\Omega)_{, i j}}{V^{0}-V^{3}}\left(p_{0}\right) .
$$

Hence, it follows from (6) that $\Omega_{, 12}\left(p_{0}\right)=W_{, 12}\left(p_{0}\right)=0$. Moreover, from (19) we have $W_{, i i}\left(p_{0}\right)=-\left(\varphi \lambda_{i} \Omega_{i, i}\right)\left(p_{0}\right)$. Hence, $Z_{, 12}^{3}\left(p_{0}\right)=0$ and it follows from (16) that

$$
Z_{, i i}^{3}\left(p_{0}\right)=\frac{c(c-1)\left(1+\lambda_{i}\right)^{2} \varphi^{2}}{Y^{0}-Y^{3}}\left(p_{0}\right)=\frac{4 c(c-1) \lambda_{2}}{\varphi^{2}\left(Y^{0}-Y^{3}\right)}\left(p_{0}\right), \quad \forall i, i=1,2 .
$$

In other words, we have

$$
Z_{, 1}^{1}\left(p_{0}\right)=Z_{, 2}^{2}\left(p_{0}\right)=c a \quad \text { and } \quad Z_{, 2}^{1}\left(p_{0}\right)=-Z_{, 1}^{2}\left(p_{0}\right)=c b,
$$

where $a=G_{, 1}^{1}\left(p_{0}\right)$ and $b=G_{, 2}^{1}\left(p_{0}\right)$. Moreover,

$$
Z_{, i}^{3}\left(p_{0}\right)=Z_{, 12}^{3}\left(p_{0}\right)=0 \quad \text { and } \quad Z_{, i i}^{3}\left(p_{0}\right)=c(c-1) \ell, \quad \text { where } \quad \ell=\frac{4 \lambda_{2}}{\varphi^{2}\left(Y^{0}-Y^{3}\right)}\left(p_{0}\right) \text {. }
$$


By considering the Taylor expansion of $Z\left(u_{1}, u_{2}\right)$ around the point $p_{0}=\left(u_{1}^{0}, u_{2}^{0}\right)$ and changing variables

$$
x=\frac{a\left(u_{1}-u_{1}^{0}\right)+b\left(u_{2}-u_{2}^{0}\right)}{a^{2}+b^{2}}, \quad y=\frac{-b\left(u_{1}-u_{1}^{0}\right)+a\left(u_{2}-u_{2}^{0}\right)}{a^{2}+b^{2}},
$$

we conclude that

$$
Z(x, y)=Z\left(p_{0}\right)+c\left(a^{2}+b^{2}\right)(x, y, 0)+\left(x^{2}+y^{2}\right)(0,0, c(c-1) \ell / 2)+R(x, y),
$$

where $\lim _{(x, y) \rightarrow p_{0}} \frac{R(x, y)}{x^{2}+y^{2}}$ is finite. Therefore, $p_{0}$ is an embedded horosphere type end (see [ET], [LR]).

\section{LAWSON CORRESPONDENCE AND DARBOUX TRANSFORMATION}

In this section, we give a new proof of the fact that the Lawson correspondence commutes with the Darboux transformation (or Ribaucour transformation for $\mathrm{cmc}$ surfaces). This result was known to Bianchi as one of his permutability theorems Bi1] (see also [HJ]). The proof given in this paper will be very useful for the applications given in section 4 and also for proving that the property of completeness is preserved by the commutativity.

Consider a simply connected surface $M$ of constant mean curvature $H$, contained in a space form $\bar{M}^{3}(k)$, with induced metric $I$ and shape operator $A$. Let $H^{\prime}$ be a real constant, $H^{\prime} \neq H$. Define an operator $A^{\prime}:=A+\left(H^{\prime}-H\right) I d$. Observe that $A^{\prime}$ is symmetric and has the same eigenvectors as $A$. Moreover, the pair $I, A^{\prime}$ satisfies the Gauss and Codazzi equations for a surface $M^{\prime}$, isometric to $M$, with constant mean curvature $H^{\prime}$, in the three-dimensional space form $\bar{M}^{\prime}\left(k^{\prime}\right)$ with constant curvature $k^{\prime}=k-\left(H^{\prime}\right)^{2}+H^{2}$. We say that $M$ and $M^{\prime}$ are related by the Lawson correspondence [La]. When $M$ is a minimal surface, $M^{\prime}$ is also referred to as a cmc cousin of $M$. In particular, minimal surfaces of $\mathbb{R}^{3}$ (resp. $S^{3}$ ) correspond to $\mathrm{cmc} 1$ in $\mathbb{H}^{3}$ (resp. $\mathbb{R}^{3}$ ).

Let $M$ be a surface of constant mean curvature $H$, with no umbilic points, contained in a three dimensional space form $\bar{M}(k)$. Consider $M^{\prime}$ to be the surface of constant mean curvature $H^{\prime} \neq H$ contained in $\bar{M}\left(k^{\prime}\right)$ that corresponds to $M$ by the Lawson correspondence, where $k^{\prime}=k-\left(H^{\prime}\right)^{2}+H^{2}$.

Let $\tilde{M} \subset \bar{M}^{3}(k)$ be a cmcH surface associated to $M$ by a Ribaucour transformation as in Theorem 1.2, with constant $c$ satisfying (7). Similarly, consider $\tilde{M}^{\prime} \subset \bar{M}^{3}\left(k^{\prime}\right)$ to be a $\mathrm{cmcH}^{\prime}$ surface associated to $M^{\prime}$ by a Ribaucour transformation with constant $c^{\prime}$ satisfying (77), where $k$ is replaced by $k^{\prime}$.

Our next result shows that $\tilde{M}^{\prime}$ and $\tilde{M}$ are related by the Lawson correpsondence; i.e., the following diagram commutes:

$$
\begin{aligned}
& M \subset \bar{M}^{3}(k) \quad \rightarrow \quad M^{\prime} \subset \bar{M}^{3}\left(k^{\prime}\right) \\
& \tilde{M} \subset \bar{M}^{3}(k) \quad \rightarrow \quad \tilde{M}^{\prime} \subset \bar{M}^{3}\left(k^{\prime}\right),
\end{aligned}
$$

where the constants $c \neq 0, c^{\prime} \neq 0$ and the solutions of the corresponding Ribaucour transformations are related as follows:

$$
c^{\prime}=c+H^{\prime}-H, \quad \Omega^{\prime}=\Omega, \quad W^{\prime}=W+\left(H^{\prime}-H\right) \Omega .
$$


Theorem 3.1. The Lawson correspondence commutes with the Darboux transformation (or Ribaucour transformation for surfaces of the same constant mean curvature).

Proof. Let $M \subset \bar{M}^{3}(k)$ and $M^{\prime} \subset \bar{M}^{3}\left(k^{\prime}\right)$ be surfaces of constant mean curvature $H$ and $H^{\prime}$, respectively, that are related by the Lawson correspondence, where $H^{\prime} \neq H$ and $k^{\prime}=k-\left(H^{\prime}\right)^{2}+H^{2}$.

Let $\tilde{M} \subset \bar{M}^{3}(k)$ and $\tilde{M}^{\prime} \subset \bar{M}^{3}\left(k^{\prime}\right)$ be surfaces of constant mean curvature $H$ and $H^{\prime}$ associated to $M$ and $M^{\prime}$ by a Ribaucour transformation as in Theorem 1.2, with constants $c$ and $c^{\prime}$ respectively satisfying (7).

Let $I$ be the induced metric on $M$ and let $-\lambda_{i}$ be the principal curvatures. It follows from (11) and (12) that the metric $\tilde{I}$ and the principal curvatures $-\tilde{\lambda}_{i}$ of the surface $\tilde{M}$ are given by

$$
\begin{gathered}
\tilde{I}=\frac{\Omega^{2}\left(H+\lambda_{i}\right)^{2}}{(W-H \Omega)^{2}} I, \quad \forall i=1,2, \\
-\tilde{\lambda}_{i}=H-\frac{(W-H \Omega)^{2}}{\Omega^{2}\left(H+\lambda_{i}\right)}, \quad i=1,2,
\end{gathered}
$$

where $\Omega$ and $W$ are solutions of (6).

Since $M^{\prime}$ corresponds to $M$ by the Lawson correspondence, its metric is $I^{\prime}=I$ and its principal curvatures are

$$
-\lambda_{i}^{\prime}=-\lambda_{i}+H^{\prime}-H
$$

Now consider $c^{\prime}, \Omega^{\prime}$ and $W^{\prime}$ defined by (24). It is easy to see that the functions $\Omega^{\prime}$ and $W^{\prime}$ satisfy the system of equations (6) for the constants $c^{\prime}$ and $k^{\prime}$. A straightforward computation also shows that the algebraic condition

$$
\Omega_{1}^{2}+\Omega_{2}^{2}+W^{2}+k \Omega^{2}=2 c \Omega(W-H \Omega)
$$

implies that

$$
\left(\Omega_{1}^{\prime}\right)^{2}+\left(\Omega_{2}^{\prime}\right)^{2}+\left(W^{\prime}\right)^{2}+k^{\prime}\left(\Omega^{\prime}\right)^{2}=2 c^{\prime} \Omega^{\prime}\left(W^{\prime}-H^{\prime} \Omega^{\prime}\right) .
$$

Therefore, these functions define the surface $\tilde{M}^{\prime}$ of constant mean curvature $H^{\prime}$ associated to $M^{\prime}$ by Ribaucour transformation with the constant $c^{\prime}$. Moreover, it follows from (11), (12), (27) and (24) that the metric of $\tilde{M}^{\prime}$ is given by

$$
\tilde{I}^{\prime}=\frac{\left(\Omega^{\prime}\right)^{2}\left(H^{\prime}+\lambda_{i}^{\prime}\right)^{2}}{\left(W^{\prime}-H^{\prime} \Omega^{\prime}\right)^{2}} I^{\prime}=\frac{\Omega^{2}\left(H+\lambda_{i}\right)^{2}}{(W-H \Omega)^{2}} I=\tilde{I},
$$

and the principal curvatures of $\tilde{M}^{\prime}$ are given by

$$
-\tilde{\lambda}_{i}^{\prime}=H^{\prime}-\frac{\left(W^{\prime}-H^{\prime} \Omega^{\prime}\right)^{2}}{\left(\Omega^{\prime}\right)^{2}\left(H^{\prime}+\lambda_{i}^{\prime}\right)}, \quad i=1,2 .
$$

We conclude from (25) and (26) that $\tilde{I}=\tilde{I}^{\prime}$ and $-\tilde{\lambda}_{i}^{\prime}=-\tilde{\lambda}_{i}+H^{\prime}-H$. Therefore, $\tilde{M}$ and $\tilde{M}^{\prime}$ are related by the Lawson correspondence.

Corollary 3.2. Let $X: U \rightarrow \bar{M}^{3}(k)$ and $X^{\prime}: U \rightarrow \bar{M}^{3}\left(k^{\prime}\right)$ be immersions related by the Lawson correspondence, i.e., $X$ and $X^{\prime}$ are isometric $\mathrm{cmcH}$ and $\mathrm{cmcH}^{\prime}$ surfaces respectively, such that $k+H^{2}=k^{\prime}+\left(H^{\prime}\right)^{2}$ and $U$ is a simply connected 
subspace of $\mathbb{R}^{2}$. Let $\widetilde{X}$ and $\widetilde{X}^{\prime}$ be the Ribaucour transformations of $X$ and $X^{\prime}$, with constants $c$ and $c^{\prime}$, respectively, given by (9). Then

a) The surfaces $\widetilde{X}$ and $\widetilde{X}^{\prime}$ are defined on the same subset of $U$.

b) The surfaces of the family $\widetilde{X}$ are complete if and only if the surfaces of $\widetilde{X}^{\prime}$ are complete.

Proof. From Theorem 1.2, we know that $\widetilde{X}$ and $\widetilde{X}^{\prime}$ are defined on the set $U$ punctured at the points that annihilate $\Omega(W-H \Omega)$ and $\Omega^{\prime}\left(W^{\prime}-H^{\prime} \Omega^{\prime}\right)$ respectively. Therefore, item a) follows from the relations (24) and b) follows from Theorem 3.1 and the fact that $\widetilde{X}$ and $\tilde{X}^{\prime}$ are isometric.

\section{Applications}

In this section, we will first show some examples of Theorem 3.1 with surfaces that are already known and then we will obtain new families of constant mean curvature surfaces by applying this theorem.

Example a). The cmc1 surfaces in $\mathbb{R}^{3}$ associated by Ribaucour transformations to the cylinder (see [CFT2]) and the minimal surfaces in $S^{3}$ associated to the Clifford torus [TW1] are related by the Lawson correspondence. In fact, we observe that the cmc1 cylinder and the Clifford torus are related by the Lawson correspondence. In order to apply Theorem 3.1, we need to consider the cylinder parametrized by

$$
X\left(u_{1}, u_{2}\right)=\left(\frac{1}{2} \cos \sqrt{2} u_{1}, \frac{1}{2} \sin \sqrt{2} u_{1},-\frac{u_{2}}{\sqrt{2}}\right),
$$

and the Clifford torus by

$$
X^{\prime}\left(u_{1}, u_{2}\right)=\frac{1}{\sqrt{2}}\left(\cos u_{1}, \sin u_{1}, \cos u_{2}, \sin u_{2}\right) .
$$

With these parametrizations, the metric of both surfaces is given by $I=\frac{1}{2}\left(d u_{1}^{2}+\right.$ $d u_{2}^{2}$ ) and the corresponding principal curvatures $-\lambda_{1}=2, \lambda_{2}=0$ and $-\lambda_{1}^{\prime}=$ $1,-\lambda_{2}^{\prime}=-1$ are related by $-\lambda_{i}^{\prime}=-\lambda_{i}-1$.

The cmc1 surfaces in $\mathbb{R}^{3}$, associated to the cylinder by the Ribaucour transformation, were obtained in [CFT2]. By considering the parametrization (28), the solutions of (6) are given by

$$
\Omega=r\left(u_{1}\right)-s\left(u_{2}\right), \quad W=2 r,
$$

where $r\left(u_{1}\right)$ and $s\left(u_{2}\right)$ satisfy the differential equations

$$
\ddot{r}-(c-2) r=0, \quad \ddot{s}+c s=0,
$$

where the dots mean derivatives. The solutions of (6) for the Clifford torus were obtained in [TW1] and they satisfy $\Omega^{\prime}=\Omega, W^{\prime}=W-\Omega, c^{\prime}=c-1$ as in Theorem 3.1 .

Our next example will consider the family of minimal surfaces in $\mathbb{R}^{3}$ associated to the catenoid by Ribaucour transformations and the cmc1 surfaces in $\mathbb{H}^{3}$ associated to the singular catenoid cousin. Later in this section, we will consider other cmc1 cousins of the catenoid. We start by solving the system of equations (13) of the Ribaucour transformation for the family of homothetic catenoids in $\mathbb{R}^{3}$ parametrized by

$$
X\left(u_{1}, u_{2}\right)=\frac{\gamma}{2}\left(\cos 2 u_{2} \cosh 2 u_{1}, \sin 2 u_{2} \cosh 2 u_{1},-2 u_{1}\right),
$$


where $\left(u_{1}, u_{2}\right) \in \mathbb{R}^{2}$. The first fundamental form and the principal curvatures $-\lambda_{i}$ are given by

$$
I=\phi\left(u_{1}, u_{2}\right)^{2}\left(d u_{1}^{2}+d u_{2}^{2}\right), \quad \lambda_{1}=-\lambda_{2}=\frac{2 \gamma}{\phi^{2}}, \quad \text { where } \phi=\gamma \cosh 2 u_{1} .
$$

Then it follows from the computations of CFT1 that the solution of the system (6) with $H=0, k=0$, for the catenoid, is given by

$$
W=\frac{1}{\phi}\left(r\left(u_{1}\right)+s\left(u_{2}\right)\right), \quad \Omega=\frac{1}{\gamma^{2}}\left(\frac{\phi r}{c}-\frac{\phi^{\prime} \dot{r}}{4 c}+\frac{\phi \gamma}{2}(r-s)\right),
$$

where $r\left(u_{1}\right)$ and $s\left(u_{2}\right)$ satisfy the differential equations

$$
\ddot{r}-4(1-c \gamma) r=\ddot{s}+4(1-c \gamma) s=0,
$$

and the initial conditions for $r$ and $s$ must satisfy

$$
\left[(\dot{r})^{2}+(\dot{s})^{2}-4(1-c \gamma)\left(r^{2}-s^{2}\right)\right]\left(u_{1}^{0}, u_{2}^{0}\right)=0 .
$$

Example b). The family of minimal surfaces in $\mathbb{R}^{3}$ associated to the catenoid $(\gamma=1)$ by Ribaucour transformations was obtained in [CFT1. Their properties in terms of planar embedded ends were extensively studied. The so-called singular catenoid cousin is the cousin of the catenoid (29) where $\gamma=1$.

The family of cmc1 surfaces in $\mathbb{H}^{3}$ associated to the singular catenoid cousin obtained in [TW2] is in correspondence as in Theorem 3.1. In fact, the singular catenoid cousin is given by

$$
X^{\prime}\left(u_{1}, u_{2}\right)=\left(\frac{1}{2}\left(\left(|u|^{2}+2\right) \phi-u_{1} \phi^{\prime}\right),-\frac{\phi^{\prime}}{2}+u_{1} \phi, u_{2} \phi, \frac{1}{2}\left(|u|^{2} \phi-u_{1} \phi^{\prime}\right)\right),
$$

where $\phi=\cosh \left(2 u_{1}\right)$ and $u=\left(u_{1}, u_{2}\right) \in \mathbb{R}^{2}$. The metric of the catenoid and its cousin is given by $I=\cosh ^{2} 2 u_{1}\left(d u_{1}^{2}+d u_{2}^{2}\right)$ and the principal curvatures are related as in the Lawson correspondence. Now consider the system (6), with $k=-1$ and constant $c^{\prime}$, for the catenoid cousin. One can see that the solution of this system obtained in [TW2] satisfies the property of Theorem 3.1 with $c^{\prime}=c+1$. It follows from (24) that the cmc1 surfaces associated to the singular catenoid cousin by the Ribaucour transformation can be parametrized by

$$
\tilde{X}^{\prime}=\left(1+\frac{\Omega}{(c+1) W}\right) X^{\prime}-\frac{1}{(c+1) W}\left(\frac{\Omega_{u_{1}} X_{u_{1}}^{\prime}}{\phi^{2}}+\frac{\Omega_{u_{2}} X_{u_{2}}^{\prime}}{\phi^{2}}-(W+\Omega) N^{\prime}\right),
$$

where $W$ and $\Omega$ are given by (31), $\gamma=1, c \neq 0, c \neq-1$ and $N^{\prime}$ is the unit vector field normal to $X^{\prime}$ given by

$$
\begin{aligned}
N^{\prime}=\left(\frac{\phi^{\prime}}{2} u_{1}-\frac{\phi}{2}|u|^{2}-\phi+\frac{1+|u|^{2}}{\phi},-\phi u_{1} \frac{\phi^{\prime}}{2}+\frac{2 u_{1}}{\phi},\right. & \frac{\left(2-\phi^{2}\right) u_{2}}{\phi}, \\
& \left.\frac{\phi^{\prime}}{2} u_{1}-\frac{\phi}{2}|u|^{2}+\frac{|u|^{2}-1}{\phi}\right) .
\end{aligned}
$$

Moreover, it follows from Theorem 2.2 that for each pair $\left(u_{1}, u_{2}\right)$ where $W$ vanishes the cmc1 surface $\tilde{X}^{\prime}$ has an embedded horosphere type end (this was already proved in [TW2]).

The minimal surfaces in $\mathbb{R}^{3}$ associated to the catenoid were extensively illustrated in CFT1. Figures 1 and 2 exhibit the singular cousin of the catenoid in the halfspace model of $\mathbb{H}^{3}$ and a cmc1 associated surface corresponding to the parameter $c=-3$ with $r=\cosh \left(4 u_{1}\right)$ and $r=\sin \left(4 u_{2}\right)$. For each pair $\left(0, u_{2}^{0}\right)$ where $u_{2}^{0}=$ 
$(n-1 / 4) \pi / 2$ and $n$ is an integer, the cmc1 surface of Figure 2 has an embedded horosphere type end.

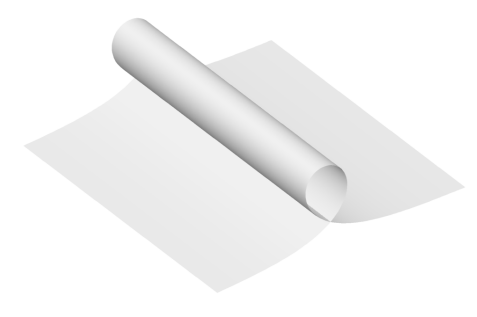

Figure 1. Singular catenoid cousin

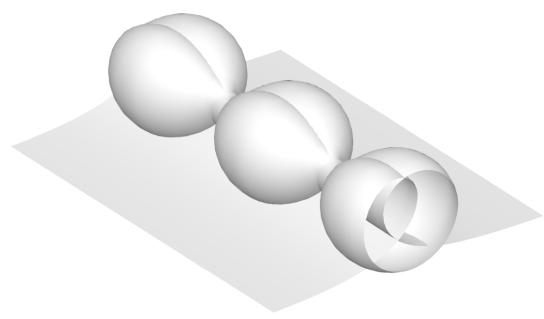

Figure 2. An associated cmc1 surface

We observe that the singular catenoid cousin parametrized by (33) (see also [MRR] is the same as the dual Enneper cousin considered in [RUY].

Example c). In this example, we want to consider the Enneper surface and its cmc1 cousin in $\mathbb{H}^{3}$, whose associated surfaces by Ribaucour transformations were investigated in [CFT1] and [TW2, respectively. In order to apply Theorem 3.1, we consider the Enneper surface in $\mathbb{R}^{3}$ parametrized by

$$
X\left(u_{1}, u_{2}\right)=-\frac{1}{24}\left(\sqrt{2}\left(u_{1}^{2}-6-3 u_{2}^{2}\right) u_{1}, \sqrt{2}\left(-u_{2}^{2}+6+3 u_{1}^{2}\right) u_{2}, 6\left(u_{1}^{2}-u_{2}^{2}\right)\right) .
$$

With this parametrization, the metric is given by $I=\varphi^{2}\left(d u_{1}^{2}+d u_{2}^{2}\right)$, where $\varphi=$ $\phi /(4 \sqrt{2}), \phi=2+u_{1}^{2}+u_{2}^{2}$ and the principal curvatures $-\lambda_{1}$ and $-\lambda_{2}$ satisfy $\lambda_{1}=$ $-\lambda_{2}=16 / \phi^{2}$. By solving the Ribaucour system of equations as in [CFT1] we get

$$
W=\frac{r\left(u_{1}\right)+s\left(u_{2}\right)}{\varphi}, \quad \text { where } \quad \ddot{s}=c s, \quad \ddot{r}=-c r
$$

and

$$
\Omega=-\frac{\sqrt{2}}{4}\left(\frac{4}{c}\left(u_{1} \dot{r}+u_{2} \dot{s}-r-s\right)+\phi(r-s)\right)
$$

We define

$$
R\left(u_{1}\right)=-4 r, \quad P\left(u_{2}\right)=4 s .
$$

Then

$$
W=\frac{\sqrt{2}(P-R)}{\phi}, \quad \ddot{R}=-c R, \ddot{P}=c P
$$

and

$$
\Omega=\frac{\sqrt{2}}{4}\left[\frac{\phi}{4}(R+P)+\frac{1}{c}\left(P-R+u_{1} \dot{R}-u_{2} \dot{P}\right)\right] .
$$


Now we consider the Enneper cousin parametrized by

$$
\begin{array}{r}
X^{\prime}\left(u_{1}, u_{2}\right)=\frac{\sqrt{2}}{2}\left(\frac{|u|^{2}+6}{4} \cosh u_{1}-u_{1} \sinh u_{1}, \frac{|u|^{2}+6}{4} \sinh u_{1}-u_{1} \cosh u_{1},\right. \\
\left.\frac{2-|u|^{2}}{4} \sin u_{2}-u_{2} \cos u_{2}, \frac{|u|^{2}-2}{4} \cos u_{2}-u_{2} \sin u_{2}\right),
\end{array}
$$

$\left(u_{1}, u_{2}\right) \in \mathbb{R}^{2}$.

Applying Theorem 3.1, it follows from (24) that the solution of the Ribaucour system for the Enneper cousin is given by

$$
\Omega^{\prime}=\frac{\sqrt{2}}{4}\left[\frac{\phi}{4}(R+P)+\frac{1}{c^{\prime}-1}\left(P-R+u_{1} \dot{R}-u_{2} \dot{P}\right)\right]
$$

and

$$
W^{\prime}=\frac{\sqrt{2}(P-R)}{\phi}+\Omega^{\prime}, \quad \ddot{R}=-\left(c^{\prime}-1\right) R, \quad \ddot{P}=\left(c^{\prime}-1\right) P,
$$

where $c^{\prime}=c+1$. This is the solution obtained in TW2.

The minimal surfaces in $\mathbb{R}^{3}$ associated to the Enneper surface were illustrated in CFT1.

Our next result will provide new 3-parameter families of cmc1 surfaces in $\mathbb{H}^{3}$ by applying Theorem 3.1 to the Bonnet family of minimal surfaces in $\mathbb{R}^{3}$ associated to the catenoid and its cmc1 cousins in $\mathbb{H}^{3}$. We observe that the minimal surfaces in $\mathbb{R}^{3}$ associated, by Ribaucour transformation, to the Bonnet family were obtained in $[\mathrm{LT}$, by considering Liouville parameters, i.e., the Weierstrass data was given by $g(z)$ and $f=1 / g_{z}$, where $z=u_{1}+i u_{2}$. Since we want to consider the family of cmc1 cousins in $\mathbb{H}^{3}$, we should consider a more general family of minimal surfaces in $\mathbb{R}^{3}$, whose fundamental forms are

$$
I=\varphi^{2} d z d \bar{z}, \quad I I=-\nu\left(d z^{2}+d \bar{z}^{2}\right)
$$

(the cousin surfaces differ according to the values of $\nu$ ); i.e., the Weierstrass data is given by $g(z)$ and $f=2 \nu / g_{z}$ and

$$
\varphi=\frac{|\nu|\left(1+|g|^{2}\right)}{\left|g_{z}\right|} .
$$

In this case, the system of equation (6) reduces to (see Proposition 2.1 in [LT]

$$
\begin{aligned}
\Omega_{z z} & =\nu(W-c \Omega)+\frac{2 \Omega_{z} \varphi_{z}}{\varphi}, \\
\Omega_{z \bar{z}} & =\frac{c \varphi^{2} W}{2} \\
W_{z} & =-\frac{2 \nu \Omega_{\bar{z}}}{\varphi^{2}} .
\end{aligned}
$$

For our next result, we consider the Bonnet family described by the Weierstrass data

$$
g(z)=e^{\mu z}, \quad f(z)=2 \frac{\nu}{\mu} e^{-\mu z}, \quad \nu \in \mathbb{R}, \mu \in \mathbb{C} .
$$

For this family,

$$
\varphi(z, \bar{z})=2\left|\frac{\nu}{\mu}\right| \cosh (\operatorname{Re}(\mu z))
$$


We observe that this family of surfaces includes the catenoid and the helicoid and their corresponding cmc1 cousins in $\mathbb{H}^{3}$.

Theorem 4.1. Let $X(z, \bar{z})$ be a parametrization of the Bonnet family of cmc1 surfaces in $\mathbb{H}^{3}$ whose Weierstrass data is given by (35). The cmc1 surfaces in $\mathbb{H}^{3}$, associated to this family by Ribaucour transformations are parametrized by

$$
\tilde{X}=\left(1+\frac{\varphi \Omega}{c F}\right) X-\frac{\varphi}{c F}(\nabla \Omega-W N), \quad c \in \mathbb{R} \backslash\{0,1\},
$$

where $\varphi$ is given by (36)

$$
\Omega=\frac{1}{c-1}\left[\frac{\varphi^{2}}{8 \nu^{2}} \Delta\left(\frac{F}{\varphi}\right)+\frac{F}{\varphi}\right], \quad W=\Omega+\frac{F}{\varphi},
$$

and

$$
F=\left\{\begin{array}{cc}
b_{1} e^{\operatorname{Re}(\beta z)}+b_{2} e^{-\operatorname{Re}(\beta z)}+b_{3} e^{i \operatorname{Im}(\beta z)}+\bar{b}_{3} e^{-i \operatorname{Im}(\beta z)}, & \text { if } \beta \neq 0, \\
b_{1} z \bar{z}+b_{3} z+\bar{b}_{3} \bar{z}+b_{2}, & \text { if } \beta=0, \\
\beta=\rho \sqrt{\mu^{2}+4 \nu(1-c)}, \quad \rho= \pm 1, &
\end{array}\right.
$$

$b_{1}, b_{2} \in \mathbb{R}, b_{3} \in \mathbb{C}$ and $b_{1} b_{2}-\left|b_{3}\right|^{2}=0$.

Proof. Consider the minimal surfaces in $\mathbb{R}^{3}$ whose Weierstrass data is given by (35). Then the fundamental forms are given by

$$
I=\varphi^{2} d z d \bar{z}, \quad I I=-\nu\left(d z^{2}+d \bar{z}^{2}\right)
$$

where $\varphi$ is given by (36). The principal curvatures are $-\lambda_{1}=-2 \nu / \varphi^{2}$ and $-\lambda_{2}=$ $\lambda_{1}$.

Observe that the metric satisfies $\varphi_{z z}=\frac{\mu^{2}}{4} \varphi$. We will denote by $\Omega_{0}, W_{0}$ the solutions of the Ribaucour transformation (34) with constant $c_{0}$, for this family of minimal surfaces. The same computations used in [LT] show that these solutions are given by

$$
\Omega_{0}=\frac{1}{c_{0}}\left[\frac{\varphi^{2}}{8 \nu^{2}} \Delta\left(\frac{F}{\varphi}\right)+\frac{F}{\varphi}\right], \quad W_{0}=\frac{F}{\varphi}
$$

where

$$
F_{z z}=\frac{\beta^{2}}{4} F, \quad \beta=\rho \sqrt{\mu^{2}-4 c_{0} \nu} .
$$

We now consider the family of cmc1 surfaces in $\mathbb{H}^{3}$, whose Weierstrass data is given by (35). Hence its fundamental forms are given by

$$
I=\varphi^{2} d z d \bar{z}, \quad I I=-\nu\left(d z^{2}+d \bar{z}^{2}\right)+I .
$$

It follows from Theorem 3.1 that the solution of the Ribaucour transformation for this family of cmc1 surfaces (cousins of the Bonnet surfaces) is given by

$$
\Omega=\Omega_{0}, \quad W-\Omega=W_{0}, \quad c-1=c_{0} .
$$

Therefore, it follows from (41) and (42) that (38) holds and

$$
F_{z z}=\frac{\beta^{2}}{4} F, \quad \beta=\rho \sqrt{\mu^{2}+4 \nu(1-c)} .
$$

This implies that (39) and (40) are satisfied. We conclude that the cmc1 surfaces in $\mathbb{H}^{3}$ associated to the cousins of the Bonnet family are parametrized by (37). 
Remark 4.2. The function $F$ given by (39), when $\beta \neq 0$, can be rewritten as (43)

$$
F(z, \bar{z})= \begin{cases}2\left|b_{3}\right|(\sigma \cosh (\operatorname{Re}(\beta z)+C)+\sin (\operatorname{Im}(\beta z)+B)) & \text { if }\left|b_{3}\right|>0, \sigma= \pm 1, \\ b_{1} e^{\operatorname{Re}(\beta z)} & \text { if }\left|b_{3}\right|=b_{2}=0, \\ b_{2} e^{-\operatorname{Re}(\beta z)} & \text { if }\left|b_{3}\right|=b_{1}=0 .\end{cases}
$$

Moreover, in the first case, i.e., $\left|b_{3}\right|>0$, any surface of the family $\widetilde{X}$ is defined for $z \in \mathbb{C} \backslash\left\{z_{k}\right\}$, where $z_{k}$ is such that

$$
\operatorname{Re}\left(\beta z_{k}\right)+C=0, \quad \operatorname{Im}\left(\beta z_{k}\right)+B=2 \pi k-\frac{\sigma \pi}{2} \quad \text { and } \quad \sigma= \pm 1 .
$$

A special case of the family of cmc1 surfaces obtained in Theorem 4.1 is given by the Ribaucour transformations of the catenoid cousins. In Example b), we considered the singular catenoid cousin that corresponds to $\mu=2$ and $\nu=-1$ and the associated cmc1 surfaces.

Excluding the singular catenoid, the general family of catenoid cousins in $\mathbb{H}^{3}$, for any $\mu, \nu$ and real $\alpha=\sqrt{\mu^{2}+4 \nu} / 2 \neq 0$ is parametrized by (see also [MRR])

$X^{\prime}\left(u_{1}, u_{2}\right)=\left(\frac{1}{8 \mu \alpha}\left((\mu+2 \alpha)^{2} \cosh \left((-\mu+2 \alpha) u_{1}\right)+(-\mu+2 \alpha)^{2} \cosh \left((\mu+2 \alpha) u_{1}\right)\right)\right.$,

$$
\begin{aligned}
& \frac{1}{4 \mu \alpha}\left(\mu^{2}-4 \alpha^{2}\right) \cosh \left(\mu u_{1}\right) \cos \left(2 \alpha u_{2}\right), \\
& \frac{1}{4 \mu \alpha}\left(\mu^{2}-4 \alpha^{2}\right) \cosh \left(\mu u_{1}\right) \sin \left(2 \alpha u_{2}\right), \\
& \left.\frac{1}{8 \mu \alpha}\left((\mu+2 \alpha)^{2} \sinh \left((-\mu+2 \alpha) u_{1}\right)+(-\mu+2 \alpha)^{2} \sinh \left((\mu+2 \alpha) u_{1}\right)\right)\right) .
\end{aligned}
$$

As a consequence of Theorem 3.1, the cmc1 surfaces in $\mathbb{H}^{3}$ associated to $X^{\prime}$ by a Ribaucour transformation are given by

$$
\tilde{X}^{\prime}=\left(1+\frac{\Omega}{(c+1) W}\right) X^{\prime}-\frac{1}{(c+1) W}\left(\frac{\Omega_{u_{1}} X_{u_{1}}^{\prime}}{\phi^{2}}+\frac{\Omega_{u_{2}} X_{u_{2}}^{\prime}}{\phi^{2}}-(W+\Omega) N^{\prime}\right),
$$

where $X^{\prime}$ is given by (45), the parameter $c \in \mathbb{R} \backslash\{0,-1\}, \phi, \Omega$ and $W$ are given by

$$
\begin{gathered}
\phi=\frac{2 \nu}{\mu} \cosh \left(\mu u_{1}\right), \quad W=\frac{1}{\phi}\left(r\left(u_{1}\right)+s\left(u_{2}\right)\right), \\
\Omega=\frac{\phi}{4 c \nu^{2}}\left[\mu^{2} r-2 \nu c(r-s)-\frac{\phi^{\prime}}{\phi} r_{u_{1}}\right],
\end{gathered}
$$

$r\left(u_{1}\right), s\left(u_{2}\right)$ satisfy the differential equations

$$
\ddot{r}-\left(\mu^{2}-4 c \nu\right) r=\ddot{s}+\left(\mu^{2}-4 c \nu\right) s=0
$$

and the initial conditions for $r$ and $s$ must satisfy

$$
\left[(\dot{r})^{2}+(\dot{s})^{2}-\left(\mu^{2}-4 c \nu\right)\left(r^{2}-s^{2}\right)\right]\left(u_{1}^{0}, u_{2}^{0}\right)=0 .
$$

As a consequence of (11) and (12), the first and second fundamental forms of the surfaces $\tilde{X}^{\prime}$ are given by

$$
\tilde{I}^{\prime}=\frac{4 \nu^{2} \Omega^{2}}{\phi^{2} W^{2}}\left(d u_{1}^{2}+d u_{2}^{2}\right), \quad \tilde{I I}^{\prime}=-\tilde{I}^{\prime}-2 \nu\left(d u_{1}^{2}-d u_{2}^{2}\right)
$$


One can see that the functions $r\left(u_{1}\right)$ and $s\left(u_{2}\right)$ are given by

$$
\begin{aligned}
& r\left(u_{1}\right)= \pm \cosh \left(\sqrt{\mu^{2}-4 c \nu} u_{1}+A\right) \\
& s\left(u_{2}\right)=\sin \left(\sqrt{\mu^{2}-4 c \nu} u_{2}+B\right) \\
& \text { if } \mu^{2}-4 c \nu>0 \text {, } \\
& r\left(u_{1}\right)=\sin \left(\sqrt{-\left(\mu^{2}-4 c \nu\right)} u_{1}+A\right) \\
& s\left(u_{2}\right)= \pm \cosh \left(\sqrt{-\left(\mu^{2}-4 c \nu\right)} u_{2}+B\right) \\
& \text { if } \mu^{2}-4 c \nu<0 \text {. }
\end{aligned}
$$

Observe that the first and second fundamental forms given by (47) are invariant under the change of variables $\left(u_{1}, u_{2}\right) \rightarrow\left(u_{1}, u_{2}+B /\left|\mu^{2}-4 c \nu\right|\right)$. Therefore, without loss of generality, we may consider $B=0$. Moreover, we observe that different values of $A$ provide distinct first and second fundamental forms. Hence the surfaces are not taken into each other by rigid motions of $\mathbb{H}^{3}$. We also observe that, by construction, the cmc1 surfaces $\tilde{X}^{\prime}$ given by (46) are associated by the Lawson correspondence to the minimal surfaces obtained from the catenoid in $\mathbb{R}^{3}$ by using the Ribaucour transformation. Examples of such minimal surfaces with distinct values of the parameter $A$ are given in [CFT1] (see Figures 5-8 there). These surfaces are certainly not taken into each other by rigid motions of $\mathbb{R}^{3}$.

There is a special class of surfaces described by $\tilde{X}^{\prime}$ which are periodic in one variable. Namely, for any value of $c$ such that

$$
\alpha \in Q, \quad \mu^{2}-4 c \nu>0, \quad \text { and } \quad \mu^{2}-4 c \nu=4 \alpha^{2} \frac{n^{2}}{m^{2}}, \quad n / m \in Q,
$$

we get a surface periodic in the variable $u_{2}$, whose period is $m \pi / \alpha$. Assuming that $n / m$ is irreducible, the integer $n$ corresponds to the number of embedded ends of horosphere type and $m$ corresponds to the geometric index of the two ends which are of catenoid type. Moreover, the surface has total curvature $-4 \pi(n+m)$.

Whenever $c$ does not satisfy (48), the surface given by (46) is not periodic in any variable and has infinitely many embedded ends of horosphere type.

As an example of such surfaces, we will consider the embedded catenoid cousin in $\mathbb{H}^{3}$ described by (45) where $\mu=2$ and $\nu=5 / 4$; hence $\alpha=3 / 2$. Figure 3 shows this catenoid cousin. In this case, for any $c \neq 0$ such that $c<4 / 5$ and $c=\frac{1}{5}\left(4-9 \frac{n^{2}}{m^{2}}\right)$, where $n / m$ is an irreducible rational number, we get a cmc1 surface which is periodic in $u_{2}$.

Figures 4, 5 and 6 exhibit the cmc1 surfaces associated to this catenoid cousin, parametrized by (46), when $n=2, n=3, n=4$ respectively and $m=1, A=0$. Figure 7 shows a view of half of the surface corresponding to $n=4$ and $m=1$ and Figure 8 shows a top view of Figure 7 .

We observe that some of the surfaces of the family may be contained in the lower half-space component of the hyperbolic space (or the external component of the ball in the Poincaré ball model). This is the case when $n=4, m=5$ and $n=8, m=9$.

Figure 9 exhibits the surface, corresponding to $n=4, m=5, A=0$, reflected to the upper half-space (or internal component of the ball). Figure 10 shows a top view of half of the surface corresponding to $u_{2}>0$.

Figure 11 exhibits the surface, corresponding to $n=8, m=9, A=0$, reflected to the upper half-space (or internal component of the ball). Figure 12 shows a top view of half of the surface, and Figure 13 shows an inner view of a section of Figure 11. 

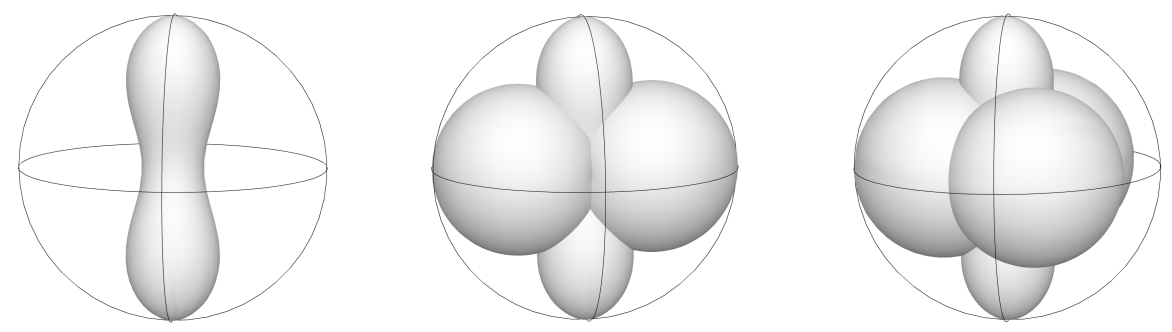

Figure 3

Figure 4

Figure 5

A cmc1 cousin of the catenoid in $\mathbb{H}^{3}$ (Figure 3) and two associated surfaces by Ribaucour transformations. The surfaces in Figure 4 and Figure 5 have, respectively, two and three embedded ends of horosphere type and two embedded ends of catenoid type.
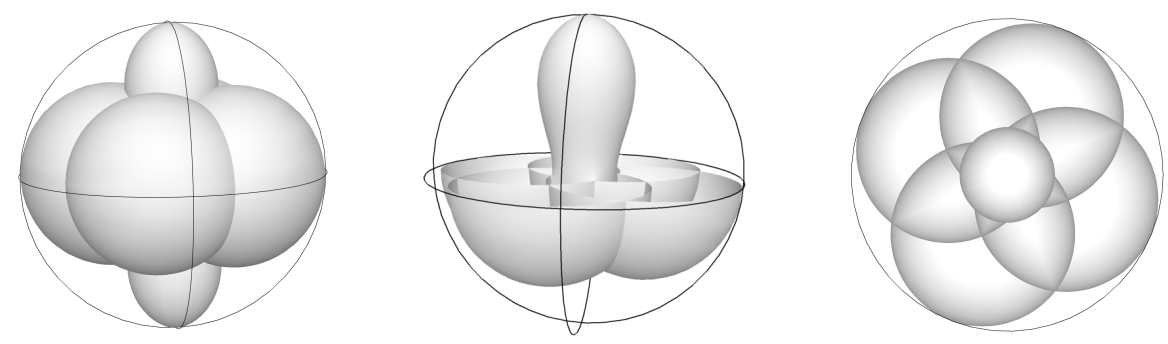

FiguRE 6

FigURE 7

FiguRE 8

A cmc1 surface in $\mathbb{H}^{3}$ associated to the catenoid cousin of Figure 3. Figure 6 has four embedded ends of horosphere type and two ends of catenoid type. Figure 7 shows a view of half of the surface. Figure 8 shows a top view of Figure 7 .

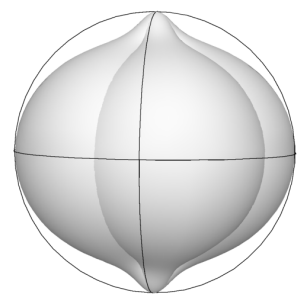

Figure 9

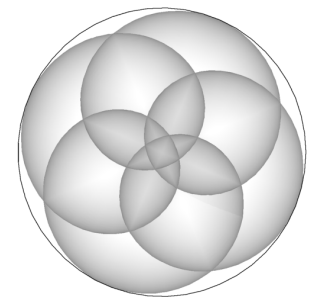

FIGURE 10

Figure 9 is a cmc1 surface in $\mathbb{H}^{3}$ associated to the catenoid cousin of Figure 3 by a Ribaucour transformation. It has 4 embedded ends of horosphere type and two ends of catenoid type of geometric index 5. Figure 10 shows a top view of half of the surface. 


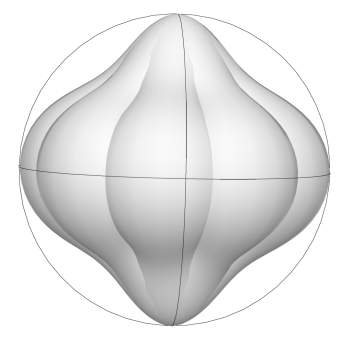

FiguRE 11. A cmc1 surface

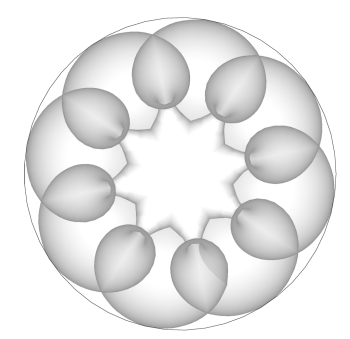

FigURE 12

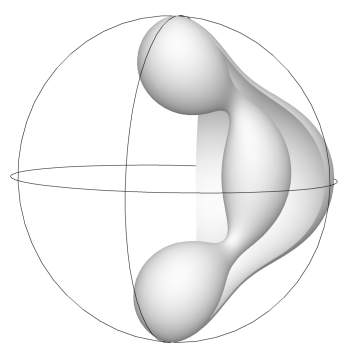

FiguRE 13

Figure 11 is a cmc1 surface in $\mathbb{H}^{3}$ associated to the catenoid cousin of Figure 3 by a Ribaucour transformation. It has 8 embedded ends of horosphere type and two ends of catenoid type of geometric index 9. Figure 12 shows a top view of half of the surface. Figure 13 shows an inner view of a section of Figure 11 .

The choices of the values for the parameter $A$, may affect the surface. See, for example, in Figure 14 and Figure 15, the surfaces corresponding to $n=8, m=9$ where $A=-1$ and $A=-1 / 2$ respectively. The surface is reflected to the upper half space (or internal component of the ball).

For any value of $c \in \mathbb{R} \backslash\{-1,0\}$ such that $c>4 / 5$ or $c<4 / 5$ and $\sqrt{4-5 c} / 3$ is not a rational number, the cmc1 surface described by (46) is not periodic in any variable and has infinitely many embedded ends of horosphere type.

A particular cmc1 surface in $\mathbb{H}^{3}$ is obtained by considering $\mu^{2}-4 c \nu=0$, i.e., in this case $c=4 / 5$. Part of this surface can be visualized in Figure 16. 

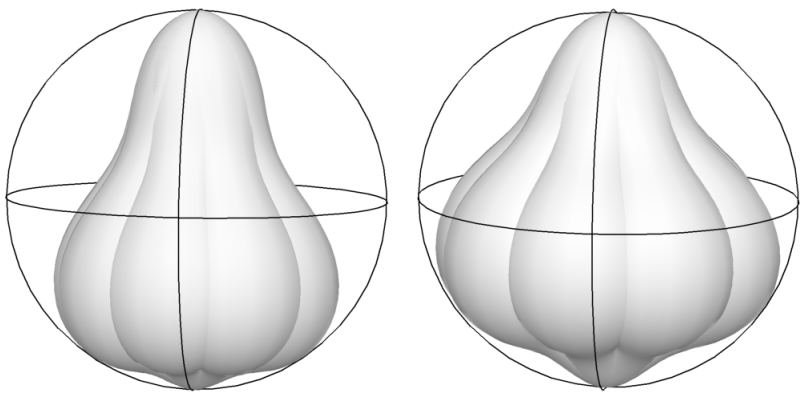

FiguRE 14

FIGURE 15

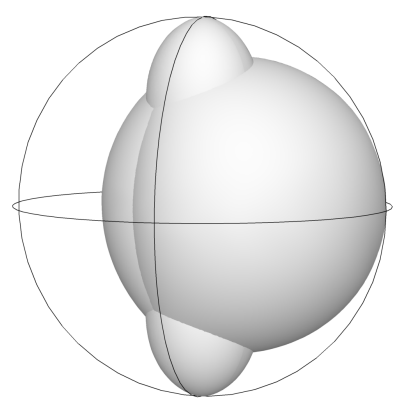

Figure 14 and Figure 15 are cmc1 surfaces in $\mathbb{H}^{3}$ associated, by a Ribaucour transformation, to the $\mathrm{cmc} 1$ cousin of the catenoid exhibited in Figure 3, with $c=\left(4-9 n^{2} / m^{2}\right) / 5$, where $n=8$, $m=9$ and $A=-1$ and $A=-1 / 2$, respectively. It has 8 embedded ends of horosphere type and two catenoid ends with geometric index 9. Figure 16 is part of a cmc1 surface in $\mathbb{H}^{3}$ associated to the cousin of the catenoid with $c=4 / 5$.

We observe that the familiy of cmc1 surfaces described by (46) contains the $\mathrm{cmc} 1$ surfaces given by Umehara-Yamada (see UY], Example 7.3). In fact, we get these surfaces by considering in (46) $\alpha=1 / 2, m=1, A=0$ and $\nu=\left(1-\mu^{2}\right) / 4$. By choosing $c=\left(\mu^{2}-n^{2}\right) /\left(1-\mu^{2}\right)$, where $n$ is an integer, we get the family of cmc1 surfaces decribed by their Weierstrass data in [UY] (their $m$ corresponds to our $n$ ).

Figure 17 shows half of the (nonembedded) catenoid cousin in the hyperbolic space, when we consider $\alpha=1 / 2$ and $\mu=5 / 2$. Applying the Ribaucour transformation to this surface, one gets a family of cmc1 surfaces depending on the parameters $c$ and $A$. In particular, when $c=\left(\mu^{2}-n^{2} / m^{2}\right) /\left(1-\mu^{2}\right)$, where $n$ and $m$ are integers and $n / m$ is irreducible, we get a surface periodic in $u_{2}$.

In Figures 18 and 19 we visualize the surface corresponding to $n=3, m=1$ and $A=0$. When $m \neq 1$ or $A \neq 0$, we get cmc1 surfaces that are distinct from the ones given in [UY]. Moreover, when $c \neq\left(\mu^{2}-n^{2} / m^{2}\right) /\left(1-\mu^{2}\right)$, the cmc1 surfaces have infinitely many embedded ends of horosphere type.

Theorem 4.3. Let $M$ be a cmc1 surface $\mathbb{H}^{3}$ whose Weierstrass data is given by (35). The surfaces $\tilde{M}$, given by Theorem 4.1 and locally associated to $M$ by a Ribaucour transformation are complete.

Proof. As we have seen in Theorem 4.1 and Remark 4.2, the parametrization of $\tilde{M}$ is given by (37), (38), where $F$ is rewritten as in (43). When $\left|b_{3}\right|=b_{1}=0$ or $\left|b_{3}\right|=b_{2}=0$ the surfaces $\widetilde{X}$ have no additional ends, so $\widetilde{X}$ is complete whenever $X$ is complete.

We now consider the case when $\left|b_{3}\right| \neq 0$. Let $\tilde{X}$ be defined by (37) on $\mathbb{C} \backslash\left\{z_{k}\right\}$, where $z_{k}$ is given by (44). In order to show that each surface of the family $\widetilde{X}$ is complete, we need to show that every divergent curve in $\widetilde{X}$ has infinite length. Such 


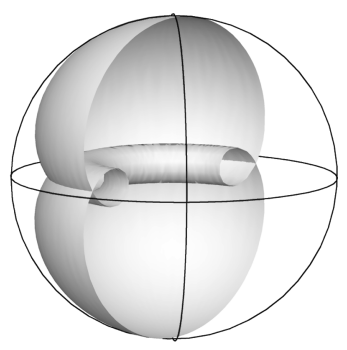

FiguRE 17

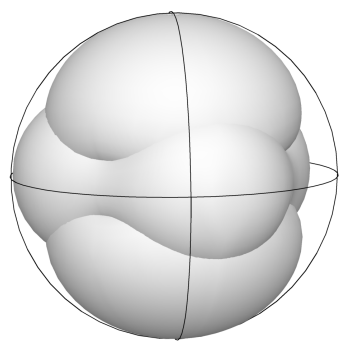

FiguRe 18

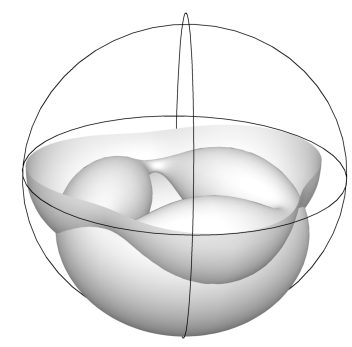

Figure 19

Figure 17 shows half of a nonembedded cmc1 cousin of the catenoid in $\mathbb{H}^{3}$, with $\alpha=1 / 2$ and $\mu=5 / 2$. Figure 18 is a cmc1 surface associated to this cousin, by a Ribaucour transformation, with $c=$ $11 / 21(n=3, m=1)$ and $A=0$. It has three embedded ends of horosphere type and two embedded ends of catenoid type. Figure 19 shows a view of half of this surface.

a curve is of the form $\widetilde{X}(z(t), \bar{z}(t)), t \in[0,1)$, where $\lim _{t \rightarrow 1} z(t)=z_{k}$ for some $k \in \mathbb{Z}$, or of the form $\widetilde{X}(z(t), \bar{z}(t)), t \in[0, \infty)$, where $\lim _{t \rightarrow+\infty}|z(t)|=+\infty$.

Claim 1. For any regular curve $z(t)=u_{1}(t)+i u_{2}(t), t \in[0,1)$, where $\lim _{t \rightarrow 1} z(t)=z_{k}$ for some $k \in \mathbb{Z}, \ell(\widetilde{X}(z(t), \bar{z}(t)))$, the length of $\widetilde{X}(z(t), \bar{z}(t))$ is infinite.

Proof of Claim 1. From (43) one has

$$
F\left(z_{k}\right)=F_{z}\left(z_{k}\right)=F_{z z}\left(z_{k}\right)=0 \quad \text { and } \quad F_{z \bar{z}}\left(z_{k}\right)=\sigma\left|b_{3} \| \beta\right|^{2},
$$

so, in the neighborhood of $z_{k}$, one has

$$
F(z, \bar{z})=\sigma\left|b_{3}\right||\beta|^{2}\left|z-z_{k}\right|^{2}+R(z, \bar{z})
$$

where

$$
\lim _{|z| \rightarrow z_{k}} \frac{R(z, \bar{z})}{\left|z-z_{k}\right|^{2}}=0
$$

By considering $\tilde{I}=\widetilde{\varphi}^{2} d z d \bar{z}$, we have

$$
\lim _{|z| \rightarrow z_{k}}\left|z-z_{k}\right|^{2} \widetilde{\varphi}=m>0, \quad \text { where } \quad \widetilde{\varphi}^{2}=\frac{4 \mu^{2} \Omega^{2}}{F^{2}}
$$

is determined by (11).

Let $z:[0,1) \rightarrow \mathbb{C} \backslash\left\{z_{k}\right\}$ be a curve, $z(t)=u_{1}(t)+i u_{2}(t)$, with $\lim _{t \rightarrow 1} z(t)=z_{k}$, for $k \in \mathbb{Z}$. Then, there exists a $t_{0} \in(0,1)$ such that $\widetilde{\varphi}(z(t))\left|z(t)-z_{k}\right|^{2} \geq m / 2$, for all 
$t \in\left[t_{0}, 1\right)$. Hence,

$$
\begin{aligned}
\ell(\tilde{X}(z(t), \bar{z}(t))) & =\lim _{u \rightarrow 1} \int_{0}^{u} \widetilde{\varphi}(z(t))\left|z^{\prime}(t)\right| d t \\
& =\lim _{u \rightarrow 1} \int_{0}^{u} \widetilde{\varphi}(z(t))\left|z(t)-z_{k}\right|^{2} \frac{\left|z^{\prime}(t)\right|}{\left|z(t)-z_{k}\right|^{2}} d t \\
& \geq \lim _{u \rightarrow 1} \frac{m}{2} \int_{t_{0}}^{u} \frac{\left|z^{\prime}(t)\right|}{\left|z(t)-z_{k}\right|^{2}} d t \\
& \geq \lim _{u \rightarrow 1} \frac{m}{2}\left|\int_{t_{0}}^{u} \frac{z^{\prime}(t)}{\left(z(t)-z_{k}\right)^{2}} d t\right|=+\infty,
\end{aligned}
$$

which concludes the proof of Claim 1.

From (11), (16) and (38) we have that

$$
\widetilde{\varphi}=\left|\frac{2 \nu \Omega}{F}\right|=\frac{|\nu| \varphi}{|c-1|}\left(\frac{1}{\nu^{2}}\left|\frac{F_{z}}{F}-\frac{\varphi_{z}}{\varphi}\right|^{2}+\frac{1}{\varphi^{2}}\right) .
$$

Since $\varphi$ is given by (36),

$$
\widetilde{\varphi} \geq \frac{\varphi}{|\nu(c-1)|}\left(\left|\frac{F_{z}}{F}\right|^{2}-\frac{\operatorname{Re}\left(\bar{\mu} F_{z}\right)}{F}+\frac{1}{4}\right) .
$$

Moreover, since $b_{1} b_{2} \neq 0$, we have

$$
\left|\frac{F_{z}}{F}\right|^{2}=\frac{|\beta|^{2}(\cosh (\operatorname{Re}(\beta z)+C)-\sin (\operatorname{Im}(\beta z)+B)}{4(\cosh (\operatorname{Re}(\beta z)+C)+\sin (\operatorname{Im}(\beta z)+B))} .
$$

We consider $\varepsilon>0, \delta>0$ and

$$
\begin{aligned}
& T=\{z \in \mathbb{C}:-\varepsilon \leq \operatorname{Re}(\beta z)+C \leq \varepsilon\}, \\
& D_{k}=\left\{z \in \mathbb{C}:-\delta+\frac{(4 k+\sigma) \pi}{2} \leq \operatorname{Im}(\beta z)+B \leq \frac{(4 k+\sigma) \pi}{2}+\delta\right\} \times T, \\
& D=\bigcup_{k \in \mathbb{Z}} D_{k}, \\
& T_{k}=\left\{z \in \mathbb{C}: \delta+\frac{(4 k+\sigma) \pi}{2} \leq \operatorname{Im}(\beta z)+B \leq-\delta+\frac{(4(k+1)+\sigma) \pi}{2}\right\} .
\end{aligned}
$$

Claim 2. There exists $\varepsilon_{1}>0, \delta_{1}>0, \rho_{1}>0$ and $L_{1}>0$ such that, for all $z \notin T$, if $|z|>L_{1}$, then $\widetilde{\varphi}(z) \geq \rho_{1}$.

Proof of Claim 2. If $|\beta|=1$, then

$$
\left|\operatorname{Re}\left(\bar{\mu} F_{z}\right)\right| \leq\left|b_{3}\right|(\cosh (\operatorname{Re}(\beta z)+C)+|\cos (\operatorname{Im}(\beta z)+B)|) ;
$$

thus

$$
\widetilde{\varphi} \geq \frac{\varphi}{4|\nu(c-1)|}\left(\frac{\cosh (\operatorname{Re}(\beta z)+C)-1}{\cosh (\operatorname{Re}(\beta z)+C)+1}\right) .
$$

Besides, one can choose $\tilde{\varepsilon}_{1}>0$ such that $\cosh (\operatorname{Re}(\beta z)+C)>2$ for all $z \notin T$. Then

$$
\widetilde{\varphi} \geq \frac{\varphi}{12|\nu(c-1)|} .
$$

From $|\beta| \neq 1$ we observe that

$$
\operatorname{Re}\left(\bar{\mu} F_{z}\right) \leq\left|\bar{\mu} F_{z}\right|=\left|F_{z}\right| .
$$

It follows from (50) that

$$
\widetilde{\varphi} \geq \frac{\varphi}{|\nu(c-1)|}\left(\left|\frac{F_{z}}{F}\right|-\frac{1}{2}\right)^{2} .
$$


Hence, we just need to prove that $\left|\frac{F_{z}}{F}\right| \neq \frac{1}{2}$. By choosing $\varepsilon_{1}>\tilde{\varepsilon}_{1}>0$ such that, for all $z \notin T, \cosh (\operatorname{Re}(\beta z)+A) \geq a>\left|\frac{|\beta|^{2}+1}{|\beta|^{2}-1}\right|$, we have that

$$
\begin{aligned}
& \left|\frac{F_{z}}{F}\right|^{2} \geq \frac{|\beta|^{2}(a-1)}{4(a+1)}>\frac{1}{4}, \quad \text { if } \quad|\beta|>1, \\
& \left|\frac{F_{z}}{F}\right|^{2} \leq \frac{|\beta|^{2}(a+1)}{4(a-1)}<\frac{1}{4}, \quad \text { if } \quad|\beta|<1,
\end{aligned}
$$

for all $z \notin T$. It follows that there exist $\varepsilon_{1}>0, \delta_{1}>0, L_{1}>0$ and $\rho_{1}>0$. such that, if $|z|>L_{1}$ and $z \notin T$, then $\widetilde{\varphi}(z) \geq r_{1}$. This concludes the proof of Claim 2 .

Claim 3. If $|\beta| \neq 1$, then there exist $\varepsilon_{2}>0, \delta_{2}>0, \rho_{2}>0$ and $L_{2}>0$ such that, for all $z \in T \backslash D$, if $|z|>L_{2}$, then $\widetilde{\varphi}(z) \geq \rho_{2}$.

Proof of Claim 3. We can choose $\delta_{2}>0$ such that, for all $z \in T \backslash D, \mid \sin (\operatorname{Im}(\beta z)+$ $B)|<b<| \frac{|\beta|^{2}-1}{|\beta|^{2}+1} \mid$. Hence

$$
\begin{aligned}
& \left|\frac{F_{z}}{F}\right|^{2} \geq \frac{|\beta|^{2}(1-b)}{4(1+b)}>\frac{1}{4}, \quad \text { if } \quad|\beta|>1, \\
& \left|\frac{F_{z}}{F}\right|^{2} \leq \frac{|\beta|^{2}(1+b)}{4(1-b)}<\frac{1}{4}, \quad \text { if } \quad|\beta|<1,
\end{aligned}
$$

for all $z \in T \backslash D$. By using (53) we conclude the proof of Claim 3.

Claim 4. Let $z(t)=u_{1}(t)+i u_{2}(t), t \in[0, \infty)$ be a divergent curve such that $|z| \rightarrow \infty$ when $t \rightarrow \infty$. Then the length $\ell(\tilde{X}(z(t)))=\infty$.

Proof of Claim 4. We consider $\varepsilon_{1}, \delta_{2}, \rho=\min \left\{\rho_{1}, \rho_{2}\right\}, L=\max \left\{L_{1}, L_{2}\right\}$ as in Claim 2 and Claim 3, and $t_{1}>0$ such that $\forall t \geq t_{1},|z(t)|>L$. If there exists $t_{2}>t_{1}$, such that $z(t) \notin T, \forall t \geq t_{2}$, then it follows from Claim 2 that $\forall t \geq t_{1}$ we have $\widetilde{\varphi}(z(t)) \geq r$; thus

$$
\ell(\widetilde{X}(z(t))) \geq \int_{t_{1}}^{\infty} r d t=\infty .
$$

On the other hand, if $|\beta| \neq 1$, and $\forall t_{2} \geq t_{1}$, there exists $t>t_{2}$, such that $z(t) \in T \backslash D$, then $z(t)$ intersects transversally an infinite number of subsets $T_{k}$. From Claim 3, we have that $\widetilde{\varphi}(z(t)) \geq \rho$ on $T_{k}$. Since the width of each $T_{k}$ is $2\left(\pi-\delta_{2}\right)$, we conclude that $\ell(\widetilde{X}(z(t)))=\infty$.

If $|\beta|=1$ and $\forall t_{2} \geq t_{1}$, there exists a $t>t_{2}$, such that $z(t) \in T \cap D$, then $z(t)$ intersects transversally an infinite number of subsets $T \cap D_{k}$. It follows from (52) and (50) that, if $z \in T \cap D$, then for all $\delta<\pi / 2$, and $\forall|z|>L$, we have $\widetilde{\varphi}(z) \geq \rho_{3}$ for some $\rho_{3}$. Since the width of each $T \cap D_{k}$ is $2 \delta$, we conclude that $\ell(\widetilde{X}(z(t)))=\infty$, which concludes the proof of Claim 4. It follows from Claim 1 and Claim 4 that $\widetilde{X}$ is complete, which concludes the proof of Theorem 4.3.

As an immediate consequence of Theorem 4.3 and Corollary 3.2 we obtain the following result.

Corollary 4.4. Each minimal surface in $\mathbb{R}^{3}$ associated by Ribaucour transformation to the Bonnet family is complete. 
Our next application will provide a 3-parameter family of cmc $H=-\sqrt{5} / 2$ immersed in the hyperbolic space $H^{3}$, by applying Theorem 3.1 to a cousin of the cylinder of $R^{3}$ with $H=1 / 2$. We start with the cylinder parametrized by

$$
X\left(u_{1}, u_{2}\right)=\left(\cos u_{2}, \sin u_{2}, u_{1}\right),
$$

and its cousin in $H^{3} \subset L^{4}$ whose parametrization is given by

$$
X^{\prime}\left(u_{1}, u_{2}\right)=\left(\frac{2}{\alpha} \cosh \frac{\alpha u_{2}}{2}, \frac{1}{\beta} \cos \left(\beta u_{1}\right), \frac{1}{\beta} \sin \left(\beta u_{1}\right), \frac{2}{\alpha} \sinh \frac{\alpha u_{2}}{2}\right),
$$

where

$$
\alpha=\sqrt{2(\sqrt{5}-1)}, \quad \beta=\frac{\alpha}{\sqrt{5}-1} .
$$

Its unit normal vector field is

$$
N^{\prime}=\left(\frac{1}{\beta} \cosh \frac{\alpha u_{2}}{2}, \frac{2}{\alpha} \cos \left(\beta u_{1}\right), \frac{2}{\alpha} \sin \left(\beta u_{1}\right),+\frac{1}{\beta} \sinh \frac{\alpha u_{2}}{2}\right) .
$$

It is easy to verify that $X$ and $X^{\prime}$ satisfy the conditions of Lawson's correspondence, since

$$
\lambda_{1}^{\prime}=\frac{2}{\sqrt{5}-1}, \quad \lambda_{2}^{\prime}=\frac{\sqrt{5}-1}{2}, \quad H^{\prime}=-\frac{\sqrt{5}}{2} .
$$

As a consequence of Theorem 3.1, we obtain

Theorem 4.5. Let $X^{\prime}\left(u_{1}, u_{2}\right)$ be the parametrization given by (55) of a surface in $H^{3} \subset L^{4}$ of constant mean curvature $H=-\sqrt{5} / 2$ and $N^{\prime}$ its unit normal vector field (57). Then,

$$
\tilde{X}^{\prime}=X^{\prime}+\frac{4}{(2 c-\sqrt{5}-1)(g-f)}\left((f+g) X^{\prime}-f_{u_{1}} X_{u_{1}}^{\prime}-g_{u_{2}} X_{u_{2}}^{\prime}-\frac{1}{2}[\sqrt{5}(f+g)+f-g] N^{\prime}\right),
$$

where $c<0$ or $c>1, c \neq(\sqrt{5}+1) / 2$ and

$$
\left\{\begin{array}{l}
f\left(u_{1}\right)= \pm \sqrt{1-c} \cosh \left(\sqrt{-c} u_{1}\right) \\
g\left(u_{2}\right)=\sqrt{-c} \sin \left(\sqrt{1-c} u_{2}\right)
\end{array} \quad \text { if } c<0\right.
$$

$$
\left\{\begin{array}{l}
f\left(u_{1}\right)=\sqrt{c-1} \sin \left(\sqrt{c} u_{1}\right) \\
g\left(u_{2}\right)= \pm \sqrt{c} \cosh \left(\sqrt{c-1} u_{2}\right)
\end{array} \quad \text { if } c>1, c \neq(\sqrt{5}+1) / 2\right.
$$

provides a 1-parameter family of complete surfaces in $H^{3}$ with the same constant mean curvature.

Proof. The surface $X^{\prime}\left(u_{1}, u_{2}\right)$ given by (55) is the cousin of the cylinder $X\left(u_{1}, u_{2}\right)$ given by (54). The solution of the Ribaucour transformation applied to the cylinder is given by

$$
\Omega=f\left(u_{1}\right)+g\left(u_{2}\right), \quad W=g\left(u_{2}\right)
$$

where $f$ and $g$ satisfy the differential equations (see [CFT2])

$$
\ddot{f}+c f=0, \quad \ddot{g}+(1-c) g=0 .
$$

It follows from Theorem 3.1 that the solution of the Ribaucour transformation applied to the cousin of the cylinder given by $X^{\prime}$ is given by

$$
\Omega^{\prime}=\Omega, \quad W^{\prime}=W-\frac{\sqrt{5}+1}{2} \Omega, \text { for } c^{\prime}=c-\frac{\sqrt{5}+1}{2},
$$


where $c^{\prime} \neq 0$. Therefore, from Theorem 1.2 we conclude that the family of surfaces in $H^{3}$, associated to $X^{\prime}$, with cmc $H=-\sqrt{5} / 2$ is given by

$$
\tilde{X}^{\prime}=X^{\prime}+\frac{4}{(2 c-\sqrt{5}-1)(2 W-\Omega)}\left(\Omega X^{\prime}-\sum_{j=1}^{2} \Omega_{u_{j}} X_{u_{j}}^{\prime}+W^{\prime} N^{\prime}\right) .
$$

If $c<0$, then

$f=a_{1} \cosh \left(\sqrt{-c} u_{1}\right)+b_{1} \sinh \left(\sqrt{-c} u_{1}\right), \quad g=a_{2} \cos \left(\sqrt{1-c} u_{2}\right)+b_{2} \sin \left(\sqrt{1-c} u_{2}\right)$,

where the initial conditions must satisfy the algebraic relation $(\dot{f})^{2}+(\dot{g})^{2}+(1-$ c) $g^{2}+c f^{2}=0$, i.e.,

$$
-c\left(a_{1}^{2}-b_{1}^{2}\right)=(1-c)\left(a_{2}^{2}+b_{2}^{2}\right)
$$

Hence,

$$
f= \pm \sqrt{a_{1}^{2}-b_{1}^{2}} \cosh \left(\sqrt{-c} u_{1}+A\right), \quad g=\sqrt{a_{2}^{2}+b_{2}^{2}} \sin \left(\sqrt{1-c} u_{2}+B\right),
$$

where

$$
\begin{aligned}
\cosh A=\frac{\left|a_{1}\right|}{\sqrt{a_{1}^{2}-b_{1}^{2}}}, & \sinh A=\frac{b_{1}}{\sqrt{a_{1}^{2}-b_{1}^{2}}}, \\
\sin B=\frac{a_{2}}{\sqrt{a_{2}^{2}+b_{2}^{2}}}, & \cos B=\frac{b_{2}}{\sqrt{a_{2}^{2}+b_{2}^{2}}} .
\end{aligned}
$$

Now it follows from the expression of $\tilde{X}^{\prime}$ and from (61) that we can consider

$$
f\left(u_{1}\right)= \pm \sqrt{1-c} \cosh \left(\sqrt{-c} u_{1}+A\right) \quad \text { and } \quad g\left(u_{2}\right)=\sqrt{-c} \sin \left(\sqrt{1-c} u_{2}+B\right) .
$$

Moreover, one can easily see that the first and second fundamental forms of the surface $\tilde{X}^{\prime}$ are invariant under the change of variables $\left(u_{1}, u_{2}\right) \rightarrow\left(u_{1}+A / \sqrt{|c|}, u_{2}+\right.$ $B / \sqrt{|1-c|})$. Hence the surfaces with different values of $A$ and $B$ are taken into each other by rigid motions of $\mathbb{H}^{3}$. Therefore, without loss of generality we may consider $A=B=0$, i.e., (60) holds.

Similar arguments for $c>1$ show that $f$ and $g$ are given by (60). Moreover, we observe that $f-g$ never vanishes. Finally, it follows from Corollary 3.2 that each constant mean curvature surface in $H^{3}$ described by the family $\tilde{X}^{\prime}$ is complete.

Remark 4.6. In Theorem 4.5, we started with the cousin of the Euclidean cylinder of radius 1 parametrized by (55). This is a cmcH surface in $\mathbb{H}^{3}$ (see Figure 20), with $H=-\sqrt{5} / 2$ and, by means of Ribaucour transformations, we obtained a family of $\mathrm{cmcH}$ surfaces in $\mathbb{H}^{3}$ given by (55) which is defined for any value of the parameter $c$, such that $c<0$ or $c>1$ and $c \neq(\sqrt{5}+1) / 2$.

This family contains a special class of surfaces which are periodic in one variable. Namely, whenever $c=(\sqrt{5}+1) n^{2} /\left(2 m^{2}\right)$ and $n / m>1$ is any rational number, we get a surface periodic in the variable $u_{1}$, whose period is $2 \pi n / \sqrt{c}$. For $c$ in the open interval $(1,(\sqrt{5}+1) / 2)$ one can also get such surfaces by choosing $c=(\sqrt{5}+$ 1) $n^{2} /\left(2 m^{2}\right)$, where $n / m$ is a rational number satisfying $(\sqrt{5}-1) / 2<n^{2} / m^{2}<1$. The integer $n$ corresponds to the number of bubbles or "sections" of the surface and $m$ corresponds to the geometric index of the two ends (see [CFT2]).

Figures 21, 22 and 23 exhibit the surfaces for $n=2, n=3, n=4$ and $m=1$, Figure 24 shows half of Figure 23, corresponding to $u_{2}>0$, and Figure 25] shows a top view of Figure 24. 


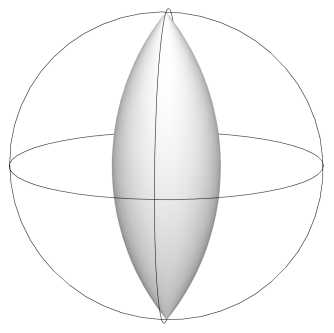

Figure 20

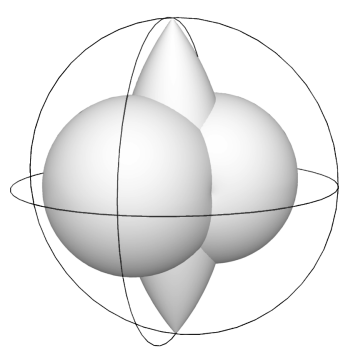

Figure 21

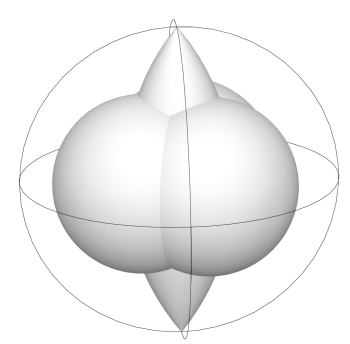

FIGURE 22

A cylinder in $\mathbb{H}^{3}$ (Figure 20) with constant mean curvature $H=$ $-\sqrt{5} / 2$ that corresponds to the Euclidean cylinder of radius one by the Lawson correspondence and two $\mathrm{cmcH}$ surfaces associated to the cylinder by Ribaucour transformations. Figure 21 has two bubbles and two cylindrical ends and Figure 22 has three bubbles and two cylindrical ends.

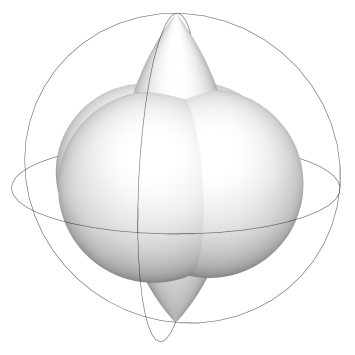

FIGURE 23

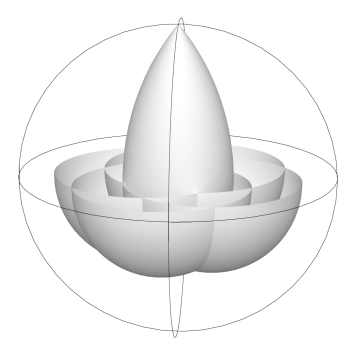

FiguRe 24

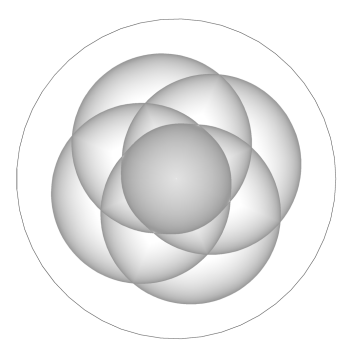

FigURE 25

A cmcH surface in $\mathbb{H}^{3}$ (Figure 23) associated to the $\mathrm{cmcH}$ cylinder by a Ribaucour transformation. It has four bubbles and two ends of cylindrical type. Figure 24 is a view of half of the surface and Figure 25 is a top view of Figure 24.

We observe that some of the surfaces of the family may be contained in the lower half-space component of the hyperbolic space (or the external component of the ball in the Poincaré ball model). This is the case when $n=4, m=5$ and $n=8, m=9$. In this case, the integer $n$ corresponds to the number of "sections" and $m$ corresponds to the geometric index of the ends.

Figure 26 exhibits the surface corresponding to $n=4, m=5$, reflected to the upper half-space (or internal component of the ball). Figure 27 shows a top view of half of the surface and Figure 28 shows part of the surface close to an end corresponding to the domain $6.95 \leq u_{2} \leq 7$.

Figure 29 exhibits the surface corresponding to $n=8, m=9$, reflected to the upper half-space (or internal component of the Poincaré ball). Figure 30 shows a top view of the lower half of the surface corresponding to $u_{2} \geq 0$ and Figure 


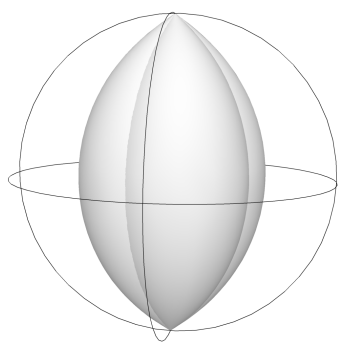

FiguRe 26

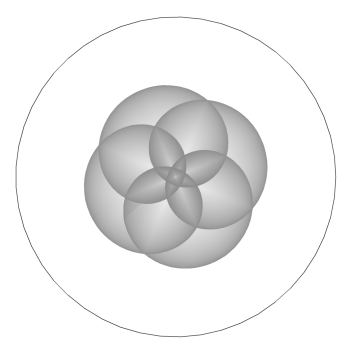

FiguRE 27

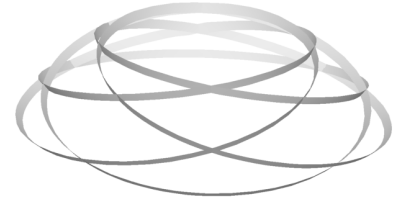

Figure 28

Figure [26 is a cmcH surface in $\mathbb{H}^{3}$ with constant mean curvature $H=-\sqrt{5} / 2$ associated to the cylinder of Figure 20 by a Ribaucour transformation. It has 4 "sections" and two cylindrical ends of geometric index 5 . Figure 27 shows a top view of the lower half of the surface, and Figure 28 shows part of Figure 26 close to an end corresponding to the domain $6.95 \leq u_{2} \leq 7$.

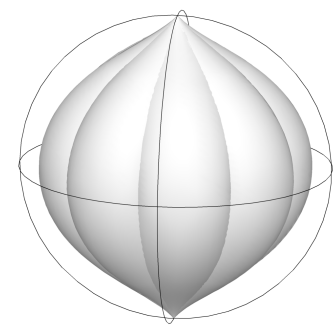

Figure 29

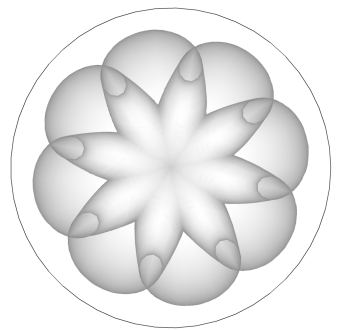

Figure 30

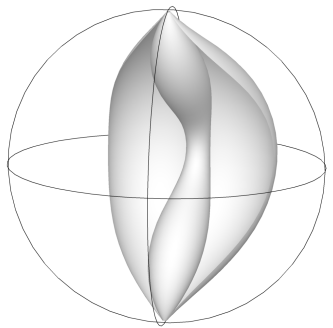

FiguRe 31

Figure 29 is a cmcH surface in $\mathbb{H}^{3}$ with constant mean curvature $H=-\sqrt{5} / 2$ associated to the $\mathrm{cmcH}$ cylinder in Figure 20 by a Ribaucour transformation. It has 8 sections and two cylindrical ends with geometric index 9. Figure 30 shows a top view of the lower half of the surface, and Figure 31]shows an inner view of part of Figure 29,

31 shows part of the internal view of the surface corresponding to the domain $0 \leq u_{1} \leq 8 \pi / \sqrt{c}$.

For other values of $c$, i.e. $c<0$ or $c>1$ and $\sqrt{c(\sqrt{5}-1) / \sqrt{2}}$ is not a rational number, the surfaces are not periodic in any variable and have infinitely many bubbles or sections. Figure 32 illustrates such a surface for $c=-3 / 2$. The figure shows the part of the surface corresponding to the domain $-1 \leq u_{1} \leq 1$ and $-7 \leq u_{2} \leq 7$. 


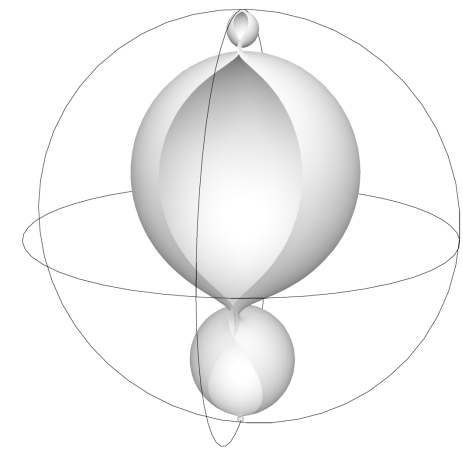

FiguRE 32

Part of a cmcH surface in $\mathbb{H}^{3}$ associated by a Ribaucour transformation to the cmcH cylinder of Figure 20. It has infinitely many bubbles in both directions approaching the boundary of the Poincaré ball model.

\section{REFERENCES}

[Bi] L. Bianchi: Le transformazioni di Ribaucour dei sistemi $\mathrm{n}^{p l i}$ ortogonali e il teorema generale di permutabilitá, Annali di Matematica (3), 27 (1918), 183-253 e (3), 28 (1919), 187-233.

[Bi1] L. Bianchi: Complementi alle ricerche sulle superficie isoterme. Ann. Mat. Pura Appl. 12 (1905), 19-54.

[Bi2] L. Bianchi: Lezioni de Geometria Differenziale Vol II, Bologna Nicola Zanichelli Editore 1927.

[Bu] F. Burstall: Isothermic surfaces: conformal geometry, Clifford algebras and integrable systems. AMS/IP Studies in Advanced Mathematics, Volume 36, 2006. MR 2222512 (2008b:53006)

[CFT1] A. V. Corro, W. Ferreira and K. Tenenblat: Minimal surfaces obtained by Ribaucour transformations. Geom. Dedicata 96 (2003), 117-150. MR1956836 (2004d:53009)

[CFT2] A. V. Corro, W. Ferreira and K. Tenenblat: Ribaucour transformations for constant mean curvature and linear Weingarten surfaces, Pacific J. Math. 212 (2003), 265-296. MR2038049(2005c:53007)

[DPW] J. Dorfmeister, F. Pedit and H. Wu: Weierstrass type representation of harmonic maps into symmetric spaces, Comm. Anal. Geom. 6 (1998), 633-668. MR.1664887(2000d:53099)

[ET] R. S. Earp and E. Toubiana: On the geometry of constant mean curvature one surfaces in hyperbolic space. Illinois J. Math. 45 (2001), 371-401. MR.1878610 (2002m:53098)

[HJ] U. Hetrich-Jeromin: Introduction to Möbius Differential Geometry. London Mathematical Society, Lecture Note Series 300. Cambridge Univ. Press, 2003. MR 2004958 (2004g:53001)

[HJP] U. Hetrich-Jeromin, F. Pedit: Remarks on the Darboux transformation of isothermic surfaces, Doc. Math 2 (1997), 313-333. MR.1487467 (99k:53006)

[Ko] S. Kobayashi: Asymptotics of ends of constant mean curvature surfaces with bubbletons. Proc. Amer. Math. Soc. 136 (2008), 1433-1443. MR2367117(2009b:53009)

[La] B. Lawson, Complete minimal surfaces in $S^{3}$. Ann. of Math. (2) 92 (1970), 335-374. MR0270280 (42:5170)

[LT] M. V. Lemes and K. Tenenblat: On Ribaucour transformations and minimal surfaces. Mat. Contemp. 29 (2005), 13-40. MR.2196777 (2006j:53007)

[LR] L. L. de Lima and W. Rossman: On the index of constant mean curvature 1 surfaces in hyperbolic space. Indiana Univ. Math. 47 (1998), 685-723. MR1647877(2000a:53010) 
[MRR] W. H. Meeks III, A. Ros, and H. Rosenberg: The Global Theory of Minimal Surfaces in Flat Spaces, Lecture Notes in Mathematics \#1775, Springer-Verlag, 2002. MR1901611 (2003i:53012)

[RUY] W. Rossman, M. Umehara and K. Yamada: Irreducible constant mean curvature 1 surfaces in hyperbolic space with positive genus. Tohoku Math. J. 49 (1997), 449-484. MR.1478909 (99a:53025)

[Sc] N. Schmitt: CMCLab software, http://www.gang.umass.edu/gallery/cmc.

[Si] H. Sievert: Über die Zentraflächen der Enneperschen Flächen konstanten krümmungsmasses, Diss. Tübingen, 1886.

[SW] I. Sterling and H. C. Wente: Existence and classification of cmc multibubbleton of finite and infinite type, Indiana Univ. Math. J. 42 (1993), 1239-1266. MR1266092 (95a:53015)

[TW1] K. Tenenblat and Q. Wang: Ribaucour Transformations for Hypersurfaces in Space Forms, Annals of Global Analysis and Geometry 29 (2006), 157-185. MR2251001 (2007e:53068)

[TW2] K. Tenenblat and Q. Wang: New constant mean curvature surfaces in the hyperbolic space. Illinois Math J. 53 (2009), 135-162. MR2584939

[UY] M. Umehara and K. Yamada: Complete Surfaces of Constant Mean Curvature 1 in the Hyperbolic 3-space. Annals of Mathematics (2) 137 (1993), 611-638. MR 1217349 (94c:53015)

Instituto de Matemática e Estatística, Universidade Federal de Goiás, 74001-970, GoiÂNIA, GO, BrazIL

E-mail address: max@mat.ufg.br

Departmento de Matemática, Universidade de Brasília, 70910-900, Brasília, DF, BRAZIL

E-mail address: roitman@mat.unb.br

Departamento de Matemática, Universidade de Brasília, 70910-900, Brasília, DF, BRAZIL

E-mail address: K.Tenenblat@mat.unb.br

Departamento de Matemática, Universidade Federal do Amazonas, Manaus, AM, BRAZIL

E-mail address: tribuzy@pq.cnpq.br 